\title{
Finding a Portfolio of Near Optimal Aggregated Solutions to Capacity Expansion Energy System Models
}

\author{
Buchholz, Stefanie; Gamst, Mette; Pisinger, David
}

Published in:

SN Operations Research Forum

Link to article, DOI:

10.1007/s43069-020-0004-y

Publication date:

2020

Document Version

Peer reviewed version

Link back to DTU Orbit

Citation (APA):

Buchholz, S., Gamst, M., \& Pisinger, D. (2020). Finding a Portfolio of Near Optimal Aggregated Solutions to Capacity Expansion Energy System Models. SN Operations Research Forum, 1, [7].

https://doi.org/10.1007/s43069-020-0004-y

\section{General rights}

Copyright and moral rights for the publications made accessible in the public portal are retained by the authors and/or other copyright owners and it is a condition of accessing publications that users recognise and abide by the legal requirements associated with these rights.

- Users may download and print one copy of any publication from the public portal for the purpose of private study or research.

- You may not further distribute the material or use it for any profit-making activity or commercial gain

- You may freely distribute the URL identifying the publication in the public portal

If you believe that this document breaches copyright please contact us providing details, and we will remove access to the work immediately and investigate your claim 
Noname manuscript No.

(will be inserted by the editor)

\title{
Finding a Portfolio of Near Optimal Aggregated Solutions to Capacity Expansion Energy System Models
}

\author{
Stefanie Buchholz • Mette Gamst • David Pisinger
}

Received: / Accepted:

\begin{abstract}
Energy system models are frequently being influenced by simplifications, assumption errors, uncertainties, incompleteness, and soft constraints which are challenging to model in a good way. In capacity expansion modeling, also the long time horizon and the high shares of renewable energies feed into the uncertainties. Consequently, a single optimal solution might not provide enough information to stand alone. Contrarily, a portfolio of different solutions, all being within an acceptance span of the system costs, would create more valuable decision support tool. This idea is known from the literature where a near optimal solution space typically is explored by introducing integer cuts that iteratively cut off solutions as they are found.

Generalizing this idea, we suggest an approach that explores the near optimal solution space by iteratively finding new solutions which are as different as possible from earlier solutions with respect to investment decisions. Our method deviates from the literature since it maximizes the difference of the found solutions rather than finding $k$ similar solutions. An advantage of this approach is that the resulting portfolio holds high diversity which creates a better basis for good decision making. Moreover, it overcomes a potential struggle of getting symmetric solutions and it strengthens the robustness arguments of the different investment decisions. Furthermore, we suggest to search for alternative solutions in an aggregated solution space whereas the original solution space typically has been used for the search in previous work. We hereby exploit the speed-up achieved through aggregation to find more solutions, and we observe that these solutions might indicate must have investments of the non-aggregated problem.

The suggested approach is tested on a case study for three different limitations on the system costs. Results show that our approach, by far outperforms the approach known from the literature when the neighborhood size exceeds $0.7 \%$. Furthermore, using our approach a portfolio of eight solutions with high diversity is found within the same time as the corresponding non-aggregated optimal solution. By looking into the different solutions, the relative importance of each unit investment is clearly identified, which potentially could be used to limit the gap between aggregated and non-aggregated solutions. Also, the portfolio in itself compensates for errors introduced by aggregation.
\end{abstract}

Keywords Near Optimal Solutions, Investment Diversification, Capacity Expansion, Energy System, Time Aggregation, Integer Cuts

S. Buchholz

Technical University of Denmark,

DTU Management Engineering,

Produktionstorvet, 424, DK-2800 Kgs. Lyngby

E-mail: stebu@dtu.dk

M. Gamst

Energinet.dk,

Tonne Kjærsvej, 65, DK-7000 Fredericia

E-mail: mga@energinet.dk

D. Pisinger

Technical University of Denmark,

DTU Management Engineering,

Produktionstorvet, 424, DK-2800 Kgs. Lyngby

E-mail: dapi@dtu.dk 


\section{Introduction}

With the climate goals of the Paris agreement trying to keep the global warming below $2^{\circ} \mathrm{C}$, a main goal of energy optimization is focused on reaching future renewable energy targets [1], and hence substantial investments in renewable energies are necessary. Which energies to use for fulfilling the goals and at which cost, are however the big decisions to be made $[1,2,3]$. As a consequence of introducing more renewable energy, the variability in production also increases significantly, hence, to find the best investments, one needs to optimize the combined long- and short-term capacity expansion energy system model $[4,5,6,7,8]$. However, the combination of a long time horizon with a fine-grained resolution, makes the resulting problems very complex to solve, and hence significant simplifications are frequently needed to make the problems solvable.

Simplifying methods both apply to the modeling and to the solution procedure. Despite of most simplifications being a necessity due to complexity challenges, some simplifications are introduced because of limitations to the optimization methodology such as neglection of real-time operations, linearization of nonlinear features, or compromisation of multi-objective aspects $[7,9]$. Frequently seen is also the omission of technicalities in the model, such as unit commitment, which compromises the solution quality [10]. The most common method to simplify the model is to reduce it in size by aggregating either the time or the spacial domain $[11,12,13,14,15]$. The general idea of domain reduction is to identify patterns in the input data and use these to reduce the data such that each pattern is represented by less variables. For capacity expansion models, this is typically seen as regions or supplying units being aggregated into one, or as a disregard of time slices not being part of an intelligent selection. Especially time aggregation has achieved a lot of recent attention with various studies comparing different techniques and experimentally quantifying the gains and errors $[16,17,18,19]$. The common conclusion is a tremendous speed-up at the cost of a slightly compromised solution quality. Moreover, due to imperfect foresight, deterministic models tend to introduce assumption errors by assuming future values for uncertain parameters in the model. Alternatively, such uncertainties are handled by introducing stochastic modeling [20,21] however, typically by compromising other detail levels, to account for the larger complexity introduced by the stochasticity [10]. Ideally the energy system models are solved to optimality using state-of-the-art solvers such as CPLEX or Gurobi [22, 23]. However, heuristic or math-heuristic approaches are used as well, potentially compromising the optimality of the solution $[24,25,26]$. Alternatively, relaxations are applied to integer variables such as investment decisions, causing yet more quality compromised solutions [22,23,27,28].

In the end, such simplifications affect the balance between solution quality and available resources. Nevertheless, all of the above mentioned references have a tendency of focusing only on the optimal solution. But with the models being dominated by uncertainties, assumptions, simplifications and imperfections due to incapabilities of modeling specific real life situations, the optimal solution might just be an approximation of the 'true' optimal solution [9,29]. Furthermore, a single optimal solution only provides a picture of the cheapest solution. It does not say anything about the importance of the decisions made nor how far from the second best solution it is, which essentially becomes highly relevant in a strategic investment framework. Imagine that the future renewable energy targets are reached in the cheapest way by making a lot of changes to the current system. However, frequently there is an $\epsilon$ more expensive solution which utilizes the current system better and therefore reaches the targets with less changes. With a cost function typically arising from both estimations and linearization, an $\epsilon$ difference in costs might be insignificant. Similarly, the single optimal solution might prioritize an investment which is not seen in any of the nearby solutions, wherefore this investment might be very sensitive to the imperfection of the modeling.

A straightforward idea is therefore to explore the near optimal solution space to find a set of solutions that respect some limit on the change in objective. This is typically achieved by introducing integer cuts which iteratively cuts away solutions from the solution space, as they are found [30]. Hereby, the $k$ best solutions are identified, all being structurally different with respect to investments. Either $k$ is a predefined number or the algorithm terminates when the change in objective exceeds a predefined limit [30]. This approach is also seen in [31] both as a standlone approach and in combination with parametric optimization. Parametric optimization is typically applied to multi-objective problems where part of the objective function is discretized and the problem is then iteratively solved for the discretized parameter [32,33]. Similarities to this idea is also seen in the proximity search method [34] where the objective is replaced by a proximity goal, ensuring the search for a new solution to be within a neighborhood around the last found solution. The idea of searching a neighborhood around a solution is that, the assumption of the maximum change in the objective 
lying within the range of uncertainties, justifies each of the solutions in the neighborhood to be equally good. A portfolio of solutions from the near optimal space therefore provide a better basis for an investor to decide on a future energy system [30]. More importantly, the different solutions provide a picture of the robustness of the different investment decisions and by that indicate the importance of each unit to the system. However, if many solutions exist in the chosen near optimal space, the resulting portfolio of solutions might not be particularly diverse, wherefore the robustness to its optimality is not stressed. Consequently we suggest to expand the approach in [30] to include a new objective, turning the old objective into a proximity constraint. To be more specific, we suggest an approach that not only searches for alternative solutions but searches for the portfolio of solutions containing highest diversity according to investments. Our approach explores the nearby optimal solution space by maximizing the differences among the found solution. Herewith, the arguments for the robustness of the decisions are strengthened while our approach provides an investor with a more fulfilling picture of the different investment possibilities within the cost limitations.

The remainder of this paper is structured as follows; Section 2 presents the suggested solution approach while Section 3 introduces a version of the near optimal solution approach from the literature. Section 4 describes a case study problem while the results are discussed in Section 5 . All observations are concluded in Section 6.

\section{Maximized Diversity Solutions}

In the following we present the suggested exploration approach in the framework of Capacity Expansion Problems (CEP), but the approach is applicable to any problem considering investment decisions. The CEP categorization covers several problems such as the Generation Expansion (GEP), demand forecasting, distribution expansion planning and transmission expansion planning [35]. All problems have the common goal of finding the optimal design of the considered part of the energy system, wherefore also combined models frequently are seen. GEPs specifically consider power plant mixes, wherefore the aim is to find an optimal mix between existing and candidate generation facilities, as well as the individual size, location and construction time. With an assumption of considering a single location with no existing capacities, the problem reduces to the decision of capacity installation of candidate units only, and this is mainly the problem considered in this paper. However, with the resent combination of long- and short-term models, both capacity mix decisions and hourly commitment of the installed units are to be decided such that the operational and investment costs are minimized [8]. Each GEP is associated to a set of constraints ensuring a match between demand and supply, balancing of the energies and further technicalities of the system to be respected. The latter applies to capacity and system related restrictions.

Let any CEP be defined as $P$ with the general problem structure as follows:

$$
\begin{array}{cl}
\min & Z=c \cdot y \\
\text { s.t. } & y \in S_{p},
\end{array}
$$

where $S_{p}$ is the set of feasible solutions. Assuming that $\hat{Z}$ is the optimized costs and $\hat{y}_{i}$ is the optimal investment strategy to the problem $P$, the suggested exploration approach aims at finding a strategy as different from $\hat{y}_{i}$ as possible, without changing $\hat{Z}$ with more than $\epsilon$. This is achieved by imposing a new objective on the problem;

$$
\max \quad \sum_{i \in I}\left|\hat{y}_{i}-y_{i}\right|
$$

while transforming the old objective into a constraint, and adding a limit to the change in system costs;

$$
\frac{|\hat{Z}-Z|}{\hat{Z}} \leq \epsilon, \quad \hat{Z}=c \cdot \hat{y}
$$

To avoid non-linearities normally imposed by the absolute value in (1), we reformulate by exploiting the variables being binary. To maximize the objective we have the following two cases;

- The known variable $\hat{y}_{i}$ is 1 : We want to minimize $y_{i}$, hence maximize $-y_{i}$

- The known variable $\hat{y}_{i}$ is 0 : We want to maximize $y_{i}$, hence maximize $+y_{i}$ 
A general formula transforming $y_{i}$ according to the above cases, is defined as follows;

$$
\left(1-2 \cdot \hat{y}_{i}\right) \cdot y_{i}
$$

Using the reformulation (3), we now define the maximized diversity problem $(Q)$ as follows:

$$
\begin{array}{ll}
\max & \sum_{i \in I}\left(1-2 \cdot \hat{y}_{i}\right) \cdot y_{i} \\
\text { s.t. } & Z=c \cdot y \\
& y \in S_{p} \\
& \frac{|\hat{Z}-Z|}{\hat{Z}} \leq \epsilon, \quad \hat{Z}=c \cdot \hat{y}
\end{array}
$$

Solving $(Q)$ now provides a solution being as different as possible to the optimal solution $\hat{y}$ with respect to investment decisions. To achieve a portfolio of maximized diversity solutions, problem $Q$ is solved in an iterative manner. We suggest two different diversity definitions, namely $Q_{\operatorname{maxmin}}$ and $Q_{\text {sum }}$. For the problem $Q_{\text {maxmin }}$ the solution in iteration $k$ is found such that it maximizes the minimum distance to the previous $k$-1 solutions. In $Q_{\text {sum }}$, the $k^{\text {th }}$ solution maximizes the sum of distances to all $k-1$ solutions. Furthermore, we introduce a cut constraint which in each iteration cuts off the solution just found, ensuring duplicates in the portfolio to be avoided. The cut constraint is defined as follow:

$$
\sum_{i} \hat{y}_{i}^{n}-1 \geq \sum_{i}\left(2 \cdot \hat{y}_{i}^{n}-1\right) \cdot y_{i}^{k} \quad \forall n \leq k-1
$$

Here, the right hand side represents a solution $n$ in terms of the new solution variable $y_{i}^{k}$ such that variables of the units included in solution $n$ have a positive sign and units not included in solution $n$ have a negative sign. The left hand side restricts this expression to be less than the sum of invested units in solution $n$ subtracted by one. Consequently, a new solution $k$ must activate at least one of the units not included in solution $n$ if the set of activated units in solution $k$ and $n$ is equal. Otherwise, the set of activated units cannot be equal, which essentially ensures that the exact same solution structure cannot appear again.

Finally, even though we maximize differences among the different investment solutions, it is still beneficial to avoid symmetric investments. This occurs in the realistic set-up where equal units are not aggregated into one. In that case, identical investment strategies might turn up in different iterations wherefore we impose symmetry constraints on the problem. Basically, such constraints are imposed on every group of identical investments. The idea is to order the investments and ensure that investments are not made in units with a higher order than the last invested unit. Formally for each technology type $t \in T$, this is defined as:

$$
y_{i_{t}}^{k} \leq y_{i_{t}-1}^{k} \quad \forall i_{t} \geq 1
$$

To summarize, the two formulations $Q_{\text {sum }}$ and $Q_{\operatorname{maxmin}}$ are as follows:

$$
\begin{aligned}
\max & \sum_{n=1}^{k-1} \sum_{i \in I}\left(1-2 \cdot \hat{y}_{i}^{n}\right) \cdot y_{i}^{k} \\
\text { s.t. } & \sum_{i} \hat{y}_{i}^{n}-1 \geq \sum_{i}\left(2 \cdot \hat{y}_{i}^{n}-1\right) \cdot y_{i}^{k} \quad \forall n \leq k-1 \\
& y_{i_{t}}^{k} \leq y_{i_{t}-1}^{k} \quad \forall i_{t} \geq 1, t \in T \\
& \frac{\left|\hat{Z}^{\{1\}}-Z^{k}\right|}{\hat{Z}^{\{1\}} \leq \epsilon, \quad \hat{Z}=c \cdot \hat{y}} \\
& Z=c \cdot y \\
& y \in S_{p}
\end{aligned}
$$

$$
\begin{aligned}
& \max M \\
& \text { s.t. } \quad M \leq \sum_{i \in I}\left(1-2 \cdot \hat{y}_{i}^{n}\right) \cdot y_{i}^{k} \quad \forall n \leq k-1 \\
& \sum_{i} \hat{y}_{i}^{n}-1 \geq \sum_{i}\left(2 \cdot \hat{y}_{i}^{n}-1\right) \cdot y_{i}^{k} \quad \forall n \leq k-1 \\
& y_{i_{t}}^{k} \leq y_{i_{t}-1}^{k} \quad \forall i_{t} \geq 1, t \in T
\end{aligned}
$$

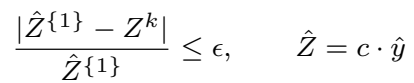

$$
\begin{aligned}
& Z=c \cdot y \\
& y \in S_{p} \\
& \left(Q_{\operatorname{maxmin}}\right)
\end{aligned}
$$

Notice that for $k=2, Q_{\text {sum }}$ and $Q_{\operatorname{maxmin}}$ become the same problem. In that case, $k_{1}$ finds the optimal solution while $k_{2}$ returns the solution with an investment strategy as different to the optimal one as possible. With $k>2$ the problem becomes more constrained for each iteration, wherefore none of the solutions found in iterations $k>k_{2}$ would exceed the investment differences obtained in $k_{2}$. We therefore refer to the solution of iteration $k_{2}$ as the Maximized Investment Different solution (MID), 
whereas all following solutions are referred to as Alternative solutions or simply Maximized Diversity Solutions (MDS), with both terminologies also possibly covering the MID. A further observation is that, since we no longer minimize costs in $Q$, the decision variables (apart from $y_{i}$ ) no longer are assigned optimized values minimizing costs. To obtain the minimized cost solution to the specific investment strategy achieved from $(Q)$, the investment strategy must be fixed in $(P)$.

\subsection{Time Aggregation Framework}

$Q_{\text {sum }}$ and $Q_{\text {maxmin }}$ can immediately be applied to problems $(P)$ of arbitrary time horizon. Nevertheless, with $(P)$ being NP-hard it is often hard to solve, and adding the suggested maximization approach, increases the solution complexity even further. Since, $Q_{\text {sum }}$ or $Q_{\text {maxmin }}$ must be solved in each iteration, we suggest to apply a problem reduction in order to reduce time usage. From the research area of capacity expansion energy system models, time aggregating simplification methods show strong time reductions without compromising the solution quality significantly $[18,19]$. Therefore, we suggest to aggregate the problem $(P)$ in each iteration $k$. For this purpose, any aggregation technique $A T Q$ can be used, see [19] for an overview and comparison of different techniques. The basic idea of time aggregation is to smartly reduce the time domain, and then solve the problem on the aggregated time domain only. The goal is to perform the reduction, such that the aggregated time series reflect essential features of the non-aggregated time series.

Since we cut off found solutions we might end up searching for nothing. To limit the consequences of this and to ensure that too much time is not wasted on unfortunate searches, we suggest to impose a time limit $T_{\max }$ on each iteration. We accept solutions found after reaching the time limit, or move to the next iteration if no solution was found.

The procedure of finding a portfolio of maximized diversity solutions is seen in Procedure 1 . The algorithm takes as input the problem $P$, the set of data series $D S$, the number of iterations $k$, the aggregation technique $A T Q$, the allowed change in cost $\epsilon$, and the time limit $T_{\max }$ for each iteration.

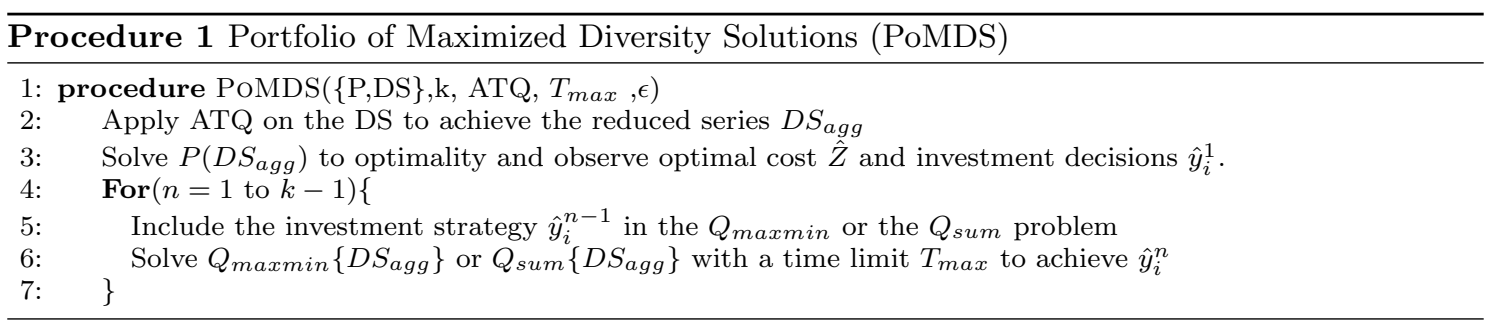

As already mentioned, using a time domain reduction approach has the advantage of regaining tractability in the problem. From the literature $[18,19]$ we see that the solutions found with a time domain reducing approach still provide high quality solutions. We hereby find a portfolio of good quality solutions in a time comparable to the time of solving the non-aggregated problem. However, despite the record of high quality solutions, there is no guarantee that the minimized costs of the portfolio of aggregated solutions stays within a $\epsilon$-range from the optimal costs, when solved in a non-aggregated framework.

\section{Near Optimal Solution Approach from Literature}

The dominating approach to exploit near optimal solutions known from the literature is an iterative cutting procedure [30,31]. The original optimization problem $(P)$ is preserved, and the $k^{\text {th }}$ solution is found by cutting away the $(k-1)$ earlier found solutions. In that sense, this procedure finds the $k$ best solutions with respect to minimized costs. We hereby argue that this approach does not have the same value as the PoMDS approach from an investment perspective. Being able to present the diversity in solutions within an acceptance range of the system costs to an investor leaves them with a wider picture for making decisions. Moreover, studying the diversity in investment choices for an uncertainty range of the system costs also provide a stronger indication of the relative importance of each investment. To investigate this claim further we suggest a comparison procedure as seen in 
Procedure 2. This algorithm finds all solutions in the $\epsilon$ neighborhood of the optimized costs, or if this amount exceeds $k$, it finds the $k$ best solutions.

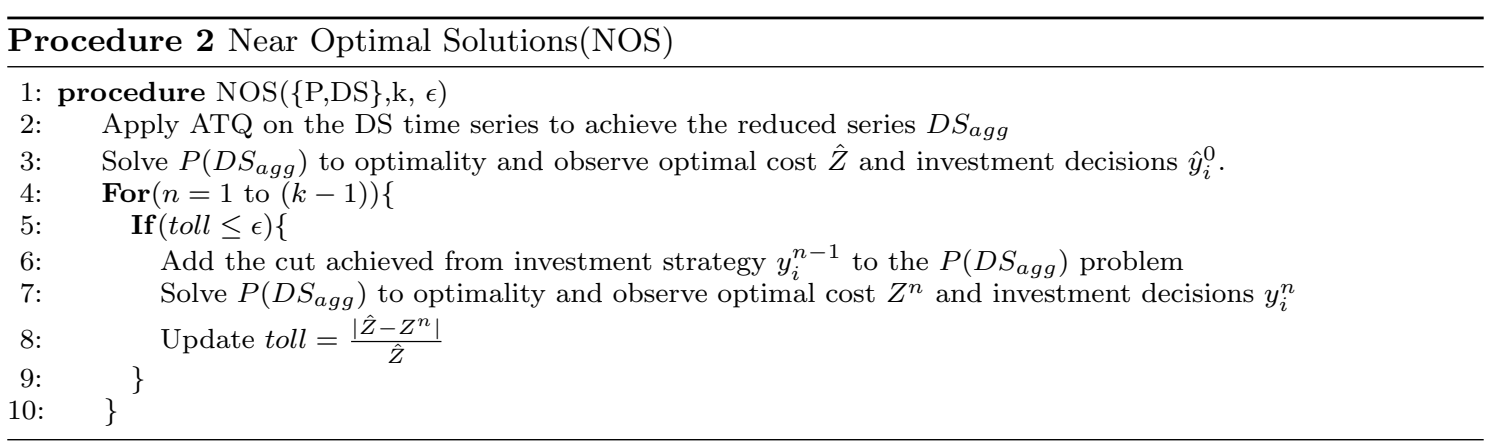

\section{Case Study}

The following case study is used to illustrate the suggested approaches. We reuse the sandbox model from [19] which is a single-region capacity expansion model featuring unit commitment. Both investment capacities and dispatch decisions are optimized such that total system costs are minimized. The mathematical formulation is described in Appendix A along with the technological input parameters assigned for this case study (Table 5). We refer to [19] for further details. Technologies available for investments are wind and PV representing Variable Renewable Energy (VRE) capacities, nuclear and coal representing base-load capacities and Open- and Combined-Cycle Gas Turbines (OCGT and CCGT) representing flexible capacities. For each technology \{Wind, PV, Nuclear, Coal, CCGT, OCGT $\}$ an initial capacity of zero is assumed. Furthermore, the same three data sets from [19] are applied here, covering demand, wind and PV time series of the Danish system from the years 2014, 2015 and 2016. These are publicly available at [36] and graphically illustrated in Figure 12 in Appendix C. For the remainder of this paper, we refer to the three problems as $P_{2014}, P_{2015}$ and $P_{2016}$.

\subsection{Aggregation Technique: Exhaustive Search (ES)}

We arbitrarily select an $A T Q$ for the case study, being the exhaustive search for an optimal set of weeks (ES). The approach is explained and tested in several papers from the literature $[13,19,37]$. With the input data series being demand (D), wind (W) and $P V(\mathrm{PV})$, we initially calculate the residual loads for each hour $h$ as: $L_{r e s}^{h}=D^{h}-W^{h} \cdot C_{W}-P V^{h} \cdot C_{P}$, where $C_{W}$ and $C_{P}$ are the assumed maximal capacities of wind and $\mathrm{PV}$ in the system, respectively. The residual load duration curve (RLDC) is created by sorting the residual loads in ascending order. We then search for the $X$ weeks which minimize the distance between the original RLDC and the RLDC achieved from the $X$ selected weeks scaled up to match the original time horizon. The search is an exhaustive search meaning that we search through all possible combinations of $X$ weeks. Algorithmically, this is achieved by a nested loop for each week selected, where each loop goes through all elements possible for selection. The ES is therefore only suitable for small values of $X$. For the purpose of this case study, we select $X=4$ meaning that we reduce the data from 52 weeks to 4 ( 8736 hours to 672 hours). The appearance of the selected weeks in the aggregated time series respect the original time chronology. The ES algorithm is outlined in Procedure 3 in Appendix B and the resulting aggregated profiles are graphically illustrated in Figure 13 and statistically represented in Table 7 both appearing in Appendix C. How the aggregation influences the size of the mathematical problem is seen in Table 6 in Appendix C.

\subsection{Approach set-up}

We apply the PoMDS algorithm to each problem $P_{2014}, P_{2015}$ and $P_{2016}$, both when using $Q_{\operatorname{maxmin}}$ and $Q_{\text {sum }}$. In each case we select $k=10$ resulting in a portfolio of ten solutions. We define the 
near optimal solution space to be the spaces allowing a $0.5 \%$, a $1 \%$ and a $5 \%$ difference in system costs respectively and we impose a two hour limitation on each iteration. Furthermore we apply the NOS algorithm to each of the problems $P_{2014}, P_{2015}$ and $P_{2016}$, searching for solutions with a maximum of $5 \%$ deviation in system costs, and a maximum of 500 solutions before termination. Also here we impose a two hour time limit on each iteration, and furthermore we terminate the search after 48 hours. All test cases are summarized in Table 1, from which it is seen that the PoMDS is represented by 18 different tests while the NOS is represented by 3 different tests. All models

Table 1: A summary of the different tests included in the case study. Here $\epsilon$ refers to the size of the neighborhood and $k$ refers to the amount of iterations. ATQ lists the applied aggregation technique.

\begin{tabular}{|c|c|c|c|c|c|c|}
\hline Test Name & Problem & Algorithm & $\mathbf{k}$ & Time Limit (sec) & $\epsilon(\%)$ & ATQ \\
\hline$M_{2014}^{0.5 \%}$ & $P_{2014}$ & $Q_{\text {maxmin }}$ & 10 & 7200 & 0.5 & $\mathrm{ES}$ \\
\hline$M_{2014}^{1 \%}$ & $P_{2014}$ & $Q_{\operatorname{maxmin}}$ & 10 & 7200 & 1 & ES \\
\hline$M_{2014}^{5 \%}$ & $P_{2014}$ & $Q_{\operatorname{maxmin}}$ & 10 & 7200 & 5 & ES \\
\hline$M_{2015}^{0.5 \%}$ & $P_{2015}$ & $Q_{\operatorname{maxmin}}$ & 10 & 7200 & 0.5 & $\mathrm{ES}$ \\
\hline$M_{2015}^{1 \%}$ & $P_{2015}$ & $Q_{\operatorname{maxmin}}$ & 10 & 7200 & 1 & $\mathrm{ES}$ \\
\hline$M_{2015}^{5 \%}$ & $P_{2015}$ & $Q_{\operatorname{maxmin}}$ & 10 & 7200 & 5 & $\mathrm{ES}$ \\
\hline$M_{2016}^{0.5 \%}$ & $P_{2016}$ & $Q_{\text {maxmin }}$ & 10 & 7200 & 0.5 & $\mathrm{ES}$ \\
\hline$M_{2016}^{1 \%}$ & $P_{2016}$ & $Q_{\operatorname{maxmin}}$ & 10 & 7200 & 1 & ES \\
\hline$M_{2016}^{5 \%}$ & $P_{2016}$ & $Q_{\operatorname{maxmin}}$ & 10 & 7200 & 5 & $\mathrm{ES}$ \\
\hline$S_{2014}^{0.5 \%}$ & $P_{2014}$ & $Q_{\text {sum }}$ & 10 & 7200 & 0.5 & ES \\
\hline$S_{2014}^{1 \%}$ & $P_{2014}$ & $Q_{\text {sum }}$ & 10 & 7200 & 1 & $\mathrm{ES}$ \\
\hline$S_{2014}^{5 \%}$ & $P_{2014}$ & $Q_{\text {sum }}$ & 10 & 7200 & 5 & ES \\
\hline$S_{2015}^{0.5 \%}$ & $P_{2015}$ & $Q_{\text {sum }}$ & 10 & 7200 & 0.5 & $\mathrm{ES}$ \\
\hline$S_{2015}^{1 \%}$ & $P_{2015}$ & $Q_{\text {sum }}$ & 10 & 7200 & 1 & ES \\
\hline$S_{2015}^{5 \%}$ & $P_{2015}$ & $Q_{\text {sum }}$ & 10 & 7200 & 5 & ES \\
\hline$S_{2016}^{0.5 \%}$ & $P_{2016}$ & $Q_{\text {sum }}$ & 10 & 7200 & 0.5 & ES \\
\hline$S_{2016}^{1 \%}$ & $P_{2016}$ & $Q_{\text {sum }}$ & 10 & 7200 & 1 & $\mathrm{ES}$ \\
\hline$S_{2016}^{5 \%}$ & $P_{2016}$ & $Q_{\text {sum }}$ & 10 & 7200 & 5 & $\mathrm{ES}$ \\
\hline Test Name & \multicolumn{2}{|c|}{ Problem } & $\mathbf{k}$ & Time Limit (sec) & $\epsilon(\%)$ & Termination (hour) \\
\hline$N^{\prime} S_{2014}$ & \multicolumn{2}{|c|}{$P_{2014}$} & 500 & 7200 & 5 & 48 \\
\hline$N O S_{2015}$ & \multicolumn{2}{|c|}{$P_{2015}$} & 500 & 7200 & 5 & 48 \\
\hline$N O S_{2016}$ & \multicolumn{2}{|c|}{$P_{2016}$} & 500 & 7200 & 5 & 48 \\
\hline
\end{tabular}

have been implemented in GAMS 24.9.2 and solved using CPLEX 12.7.1.0 with standard settings for MIPs and a $0.01 \%$ optimality gap. All experimental runs have been performed on a server with a node configuration of 2x Intel Xeon Processor 2650v4 (12 core, 2.20GHz) and $256 \mathrm{~GB}$ RAM. For each run a single core is used and $80 \mathrm{~GB}$ of memory is allocated. Despite the allocation of solution resources, different server loads might still influence the solution times of the runs.

\section{Results}

In the following section, we present the results from our case study. In relation to the suggested methods, the most essential results are the degree of diversity among investment strategies achieved for the two methods $Q_{\text {maxmin }}$ and $Q_{\text {sum }}$. Furthermore, also the balance between the solution time and degree of solution diversity is of interest, as this illuminates the benefits of the PoMDS algorithm compared to the NOS approach. We also look into the diversity of objective values achieved both in the aggregated and non-aggregated case. This is especially relevant for the question whether the nonaggregated objective value stays within the neighborhood of the optimal solution value. Moreover, a consequence of applying the diversity search to the aggregated solution space, is that the resulting investment strategies might turn up infeasible to the non-aggregated solution space, wherefore an analysis of the relation between the non-aggregated and the aggregated system costs gives relevant 
insight to the applicability of the PoMDS approach. Lastly, as we solve each iteration with a fixed time limit and a fixed amount of iterations, hence it is also important to look into how these parameter values affect the results. With the interesting question of how much an investment strategy can deviate from the optimal one without exceeding the costs limitations, we structure the following section by first analyzing the most diverse solutions of each PoMDS configuration (the MIDs) followed by an analysis of the full solution portfolios.

\subsection{Maximized Investment Different Solution}

One of the key messages of the PoMDS algorithm is to explore how different an investment strategy one could make without increasing the overall system costs too much. Therefore, the iteration with the most diverse solution is identified and are, together with the corresponding solution time and value, listed in columns 2-4 in Table 2. As expected from the definition of the PoMDS algorithm,

Table 2: Overview and specifics of the solution being most different to the optimal solution both in relation to investment decisions and in relation to the aggregated and non-aggregated system costs. Also, the degree of diversity added per solution is seen. "It." refers to the iteration of the PoMDS in which the specific solution was found. Since one solution is found in each iteration, "It." basically indicates when the specified solutions appears in the portfolio.

\begin{tabular}{|c|c|c|c|c|c|c|c|c|}
\hline & & ax. Inv & ment Diff. & & Maximum cl & hange in Syste & m Cost & Avg. Investment \\
\hline Name & It. & Value & Time & It. (Agg.) & Value (Agg.) & It. (non-Agg.) & Value (non-Agg.) & Differences (per sol.) \\
\hline$M_{2014}^{0.5 \%}$ & 2 & 4 & 1075 & 2 & 0.497 & 7 & 0.666 & 5.5 \\
\hline$M_{2014}^{1 \%}$ & 2 & 7 & 882 & 7 & 0.966 & 9 & 0.859 & 1.1 \\
\hline$M_{2014}^{5 \%}$ & 2 & 12 & 824 & 3 & 5.000 & 2 & 3.822 & 4.0 \\
\hline$M_{2015}^{0.5 \%}$ & 2 & 3 & 1345 & 7 & 0.494 & 7 & 0.628 & 3.3 \\
\hline$M_{2015}^{1 \%}$ & 2 & 6 & 1171 & 2 & 0.997 & 4 & 1.085 & 3.3 \\
\hline$M_{2015}^{5 \%}$ & 2 & 11 & 268 & 10 & 4.320 & 6 & 4.298 & 2.0 \\
\hline$M_{2016}^{0.5 \%}$ & 2 & 4 & 826 & 2 & 0.486 & 9 & 0.024 & 3.0 \\
\hline$M_{2016}^{1 \%}$ & 2 & 7 & 831 & 2 & 0.988 & 5 & 0.366 & 2.9 \\
\hline$M_{2016}^{5 \%}$ & 2 & 12 & 316 & 3 & 4.908 & 9 & 3.630 & 2.0 \\
\hline$S_{2014}^{0.5 \%}$ & 2 & 4 & 1107 & 2 & 0.497 & 10 & 0.399 & 9.1 \\
\hline$S_{2014}^{1 \%}$ & 2 & 7 & 839 & 5 & 0.966 & 4 & 0.701 & 4.3 \\
\hline$S_{2014}^{5 \%}$ & 2 & 12 & 797 & 6 & 4.960 & 8 & 8.074 & 2.8 \\
\hline$S_{2015}^{0.5 \%}$ & 2 & 3 & 2241 & 7 & 0.494 & 7 & 0.628 & 7.0 \\
\hline$S_{2015}^{1 \%}$ & 2 & 6 & 1201 & 2 & 0.997 & 4 & 1.085 & 10.3 \\
\hline$S_{2015}^{5 \%}$ & 2 & 11 & 239 & 7 & 4.870 & 7 & 4.990 & 4.3 \\
\hline$S_{2016}^{0.5 \%}$ & 2 & 4 & 856 & 2 & 0.486 & 5 & 0.024 & 3.4 \\
\hline$S_{2016}^{1 \%}$ & 2 & 7 & 898 & 8 & 0.998 & 9 & 0.369 & 3.9 \\
\hline$S_{2016}^{5 \%}$ & 2 & 12 & 295 & 8 & 4.929 & 8 & 4.485 & 5.2 \\
\hline
\end{tabular}

the most diverse solution is found as the first solution after the optimal one (iteration 2). Since the two approaches, $Q_{\text {maxmin }}$ and $Q_{\text {sum }}$, are identical in the first two iterations, they obtain the same value of unit investment differences. Therefore, also similar solution times are seen for the two approaches, but since each solution time only covers a single run, deviations occur. Nevertheless, a clear tendency of larger neighborhoods resulting in more diverse solutions combined with smaller solutions times are seen (for a graphical illustration, see Figure 17 in Appendix D). In many cases, the $5 \%$ neighborhood finds solutions, which not only are three times more diverse compared to solutions of the $0.5 \%$ neighborhood but also are found more than two times faster. On average, the most diversified solution is found in $1242 \mathrm{~s}$ in the $0.5 \%$ neighborhood, $970 \mathrm{~s}$ in the $1 \%$ neighborhood and $457 \mathrm{~s}$ in the $5 \%$ neighborhood. These average solution times suggest a decrease in solution time of approximately 130s for each factor the neighborhood is enlarged. 


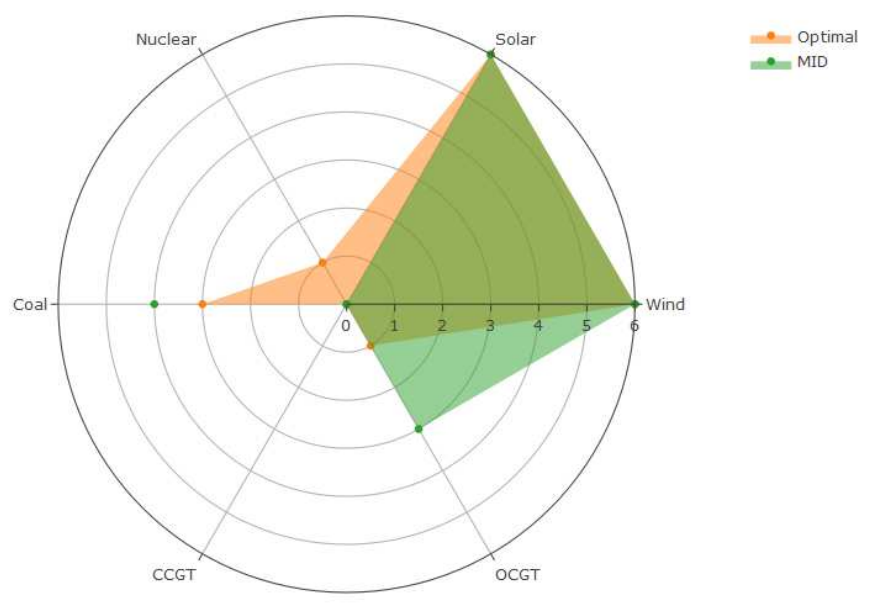

(a) In the $0.5 \%$ neighborhood.

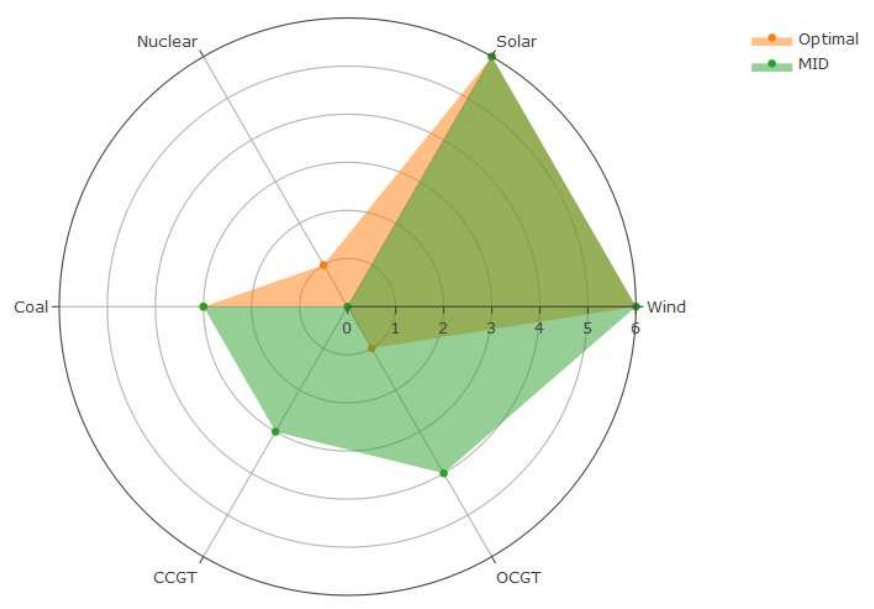

(b) In the $1 \%$ neighborhood.

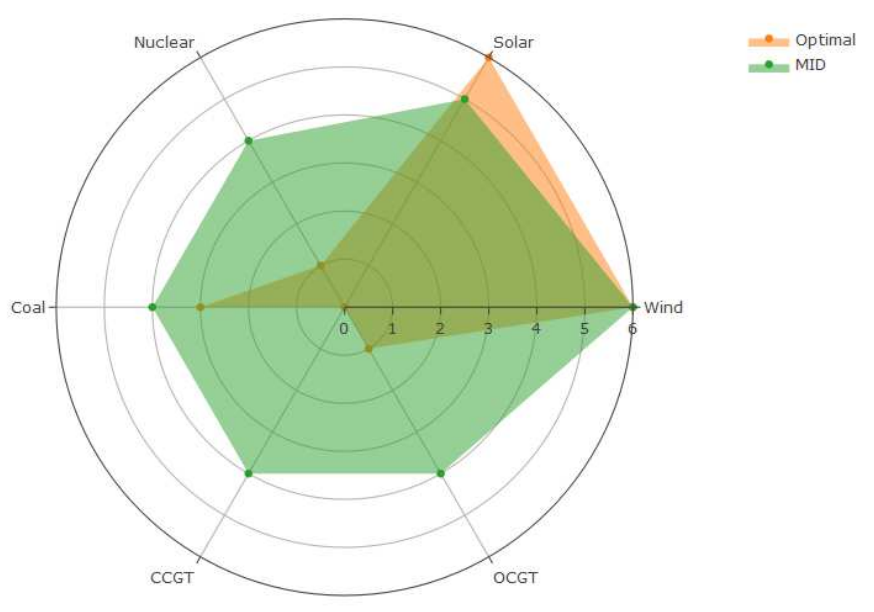

(c) In the $5 \%$ neighborhood.

Fig. 1: Illustration of the difference between the Optimal and the Maximized Investment Different (MID) solution for each of the three neighborhoods. Each graph represent results of problem $P_{2014}$. 
To emphasize on the degree of diversity in the solutions, it is seen that $43 \%$ of the investment decisions in the optimal solutions are changed in the $5 \%$ neighborhood, whereas this change is $25 \%$ in the $1 \%$ neighborhood and $14 \%$ in the $0.5 \%$ neighborhood. An illustrative way of presenting this is seen in Figure 1 (solutions related to $P_{2014}$ ), which also indicates how the investment strategy is expanded as the neighborhood is enlarged. In general, since more diversified solutions are found for larger neighborhoods, it indicates that the constraints limiting the change in system costs indeed are binding. In the framework of this case study, the gas units seem to be activated at first, followed by the coal units. Lastly, the nuclear units are activated, in which case some of the otherwise consistent renewable energy units are deactivated. However, recall that, in the maximization framework system costs are no longer minimized, wherefore this unit priority might not be the optimal one. Nevertheless, we argue that as the solution still have to be near optimal, the priority might be near optimal as well.

\subsubsection{Change in system costs}

To see how the diversity in investments relates to the deviation in system costs, the solution with the highest deviation in system costs is identified both within the aggregated and within the nonaggregated solution space (columns $5-8$ in Table 2). For the aggregated solution space, maximum investment difference and maximum system costs appear in the same solution in seven out of the 18 tests, of which none relates to the largest neighborhood. Contrarily, the maximum non-aggregated system costs only appear for one out of the 18 MID solutions. However, this solution does not also share the maximum aggregated system costs. Specific solutions covering the maximum change in both aggregated and non-aggregated system costs are seen for four of the 18 tests. It can therefore be concluded that the solution providing maximum difference to the optimal solution according to investment decisions does not necessarily lead to a binding constraint on the change in system costs. However, since different solutions are obtained for the three neighborhoods, it is known that the increase in neighborhoods are exploited in each case. From Table 2 it is seen that frequently smaller changes are observed in the non-aggregated system costs compared to in the aggregated system costs, which could be a consequence of aggregation techniques having a tendency of overestimating systems costs $[19,38,39,40]$. Another consequence introduced by aggregation appears in the maximum change of non-aggregated system costs as these in some cases exceeds the neighborhood limit. This is seen in five out of the these 18 solutions, with the largest violation being $3 \%$ (in a $5 \%$ neighborhood). Notice that, when considering the full portfolios (all 180 runs) slightly more iterations violating the neighborhood limit are seen, which is discussed in Section 5.2 together with other irregularities appearing in the runs.

Lastly, to initiate the consideration of the whole portfolio, we look into the overall degree of diversity according to investments. This is measured as the difference between each solution of the portfolio to the optimal one. Due to the imposed time limit, iterations might terminate without solutions (see Section 5.2), wherefore each portfolio not necessarily includes ten solutions. We therefore study the portfolio diversity per solution which is seen in column nine in Table 2. In general, more diversity per included solution is created in the $Q_{\text {sum }}$ approach compared to in the $Q_{\text {maxmin }}$ approach. This is to be expected due to the objective being different in the two approaches. Nevertheless, for both approaches, the degree of diversity added per solution does not seem to have a relation to the size of the neighborhood.

\subsection{Irregular Runs}

In achieving a portfolio of solutions some deviational situations might occur. Based on our test results no such situations were observed in either of the two first iterations wherefore the solutions with the most different investment strategy in every case were found without problems. However, increasing the portfolio size to include more than two solutions might introduce some irregularities. We introduce the term regular run for a run if no irregularities have occurred and irregular run if at least one irregularity occurs. We define five different types of irregularities as follows:

$I R_{1}$ : If a run is terminated due to the time limit and no solution has been found

$I R_{2}$ : If a run is terminated due to the time limit and the best solution found so far is returned

$I R_{3}$ : If the investment strategy leads to infeasibility in the aggregated solution space

$I R_{4}$ : If the investment strategy leads to infeasibility in the non-aggregated solution space 
$I R_{5}:$ If the non-aggregated system costs deviates from the optimal costs with more than $\{0.5 \%, 1 \%, 5 \%\}$

Notice that, some of the defined irregularities might overlap. An $I R_{1}$ always prevent any other irregularity to occur, like $I R_{4}$ prevents $I R_{5}$. Contrarily, $I R_{2}$ likely causes either $I R_{3}, I R_{4}$ or $I R_{5}$ to happen as well, and similarly $I R_{3}$ can overlap with both $I R_{4}$ and $I R_{5}$. Therefore the total amount of irregular runs deviates from the sum of the numbers seen in Figure 2.

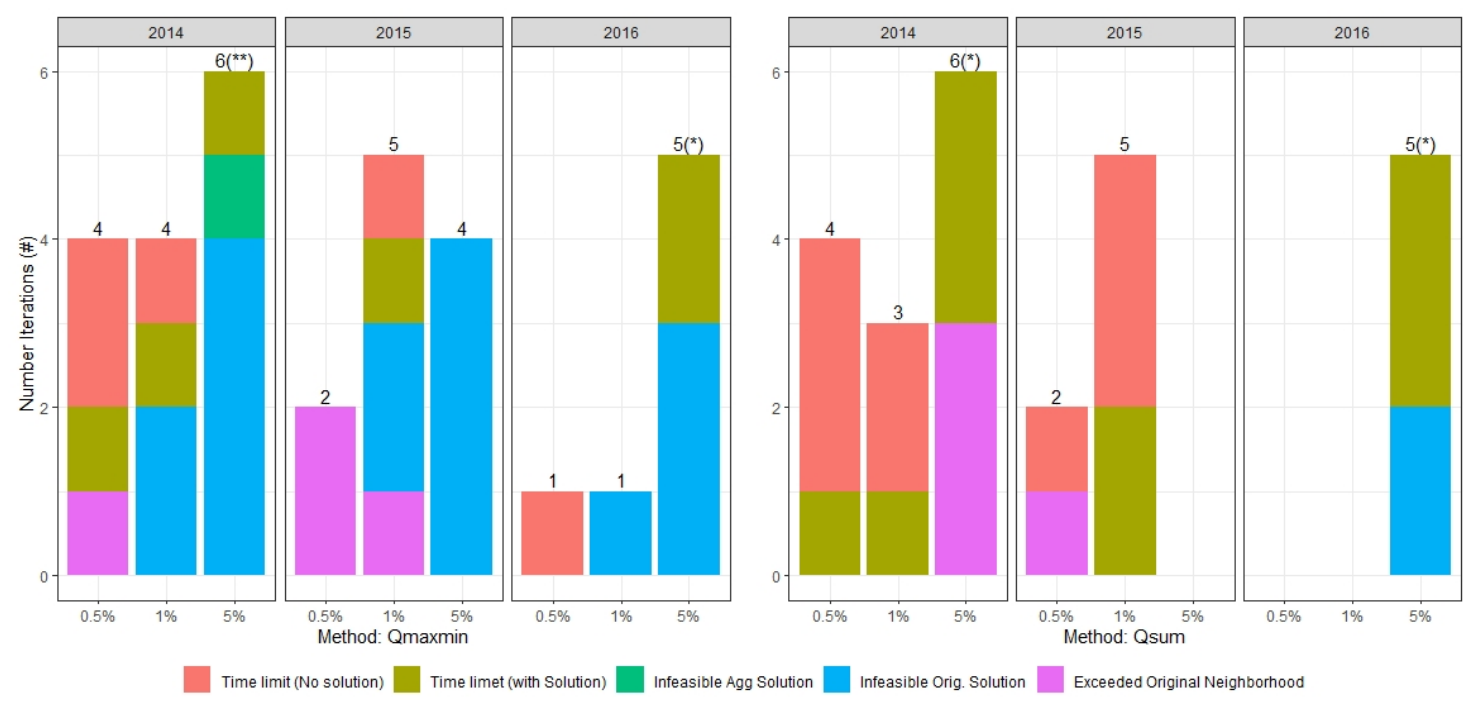

Fig. 2: Irregular runs for each method, where each bar covers the irregularities observed for the ten iterations of the specified method. A run is irregular if it exceeds the time limit, in which case either no solution has been found or the best solution found so far is returned. Furthermore, irregular runs also appear if the found investment strategy is infeasible either according to the aggregated or the non-aggregated problem. Lastly, non-aggregated system costs deviating with more than $x \%$ from the optimal costs also causes the run to be irregular. The number on top of the bars summarizes the total amount of irregularities occurring among the runs associated to the specified method. The amount of stars following these numbers indicate the overlapping irregularities (hence subtracting the amount of starts from the number results in amount of irregular iterations.)

We observe 52 irregular runs out of the 180 performed. Of these, five runs contain multiple irregularities, where the majority is the $I R_{2}$ irregularity (termination with best solution due to time limit) causing either a $I R_{4}$ or a $I R_{5}$ (infeasible solution in non-aggregated solution space and nonaggregated objective value exceeding the limitation). A single run contains the combination of a $I R_{3}$ and a $I R_{4}$, meaning that the investment strategy is infeasible both according to the aggregated and to the non-aggregated solution space. This run is associated with a $5 \%$ neighborhood, which illustrates the risk of looking into too large neighborhoods. In fact, all cases of overlapping irregularities occur in the runs related to a $5 \%$ neighborhood, (see Figure 2 where the $\left(^{*}\right)$ indicates the single occurrences), which also is the neighborhood with the highest amount of irregularities. The dominating type of irregularity is the infeasibility in the non-aggregated solution space, which is a irregularity never occuring in the $0.5 \%$ neighborhood. On the other hand, the benefit of the $5 \%$ neighborhood is that no iterations terminate without a solution, whereas this is the dominating irregularity of both the $0.5 \%$ and the $1 \%$ neighborhood. Furthermore, from Figure 2 it is seen that slightly more irregularities occur in the runs related to the $Q_{\text {maxmin }}$ approach compared to those related to the $Q_{\text {sum }}$ approach. The irregularities of the $Q_{\text {maxmin }}$ runs frequently are infeasibility in the non-aggregated solution space, while the $Q_{\text {sum }}$ runs are more affected by the time limit termination both with and without solutions returned. Naturally, the amount of irregularities seems to be related to the specific problems with (in this case) $P_{2014}$ causing the most irregular runs and $P_{2016}$ the least irregular runs. 


\subsection{Portfolio Analysis}

Now, considering portfolios rather than single solutions, the aim of the PoMDS algorithm is to explore the near optimal solution space in the search for a set of different investment strategies. Therefore, we again look into the distance between the obtained investment strategies to the optimal ones, but now for each solution contained in the portfolio. These results are seen in Figure 3, where each bar account for the accumulated diversity of the iterations for each method (a portfolio). The number on
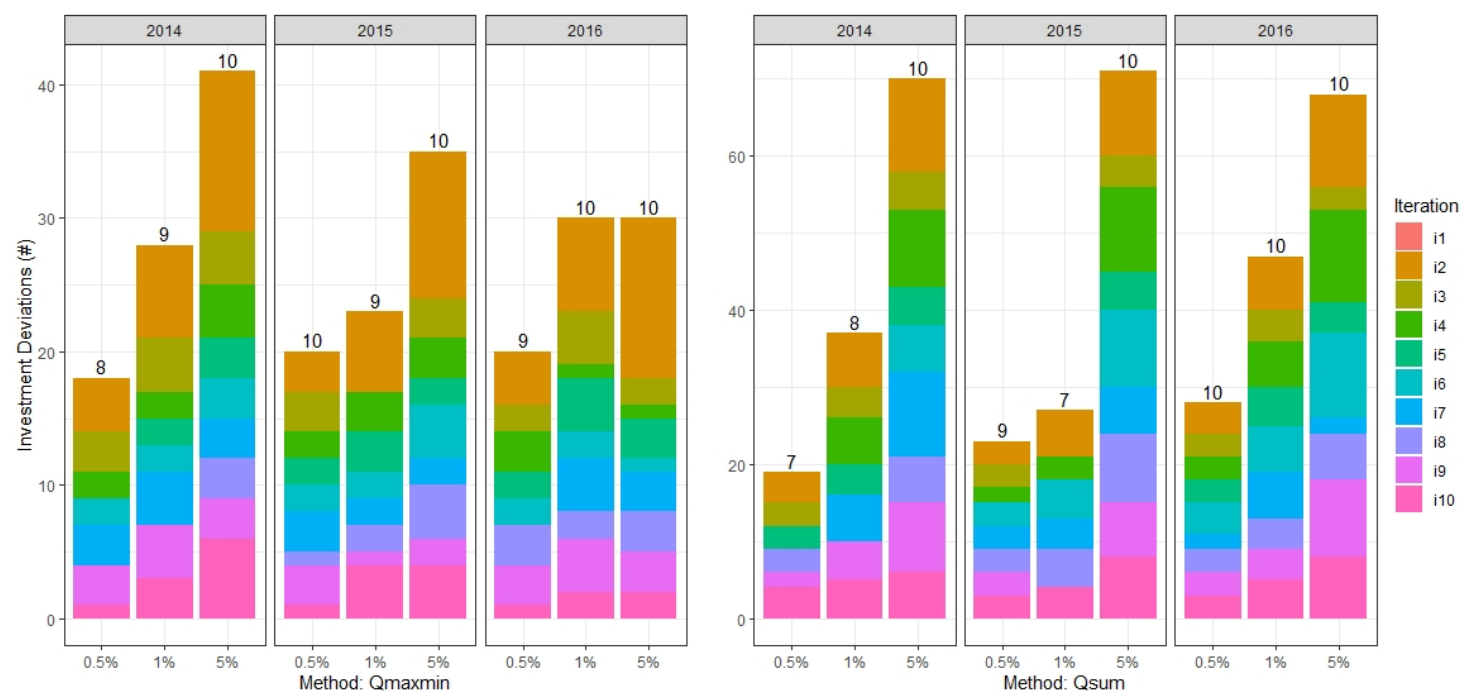

Fig. 3: Diversity in the investment strategies illustrated for each method according to iteration runs. Diversity is measured as the amount of single unit investments, which differentiates iteration run 1 to all other iteration runs. The number on the top of the bars indicates the amount of runs terminated with a solution. Since iteration $i_{1}$ finds the optimal solution the difference is 0 , wherefore it is not represented in the stacked bar.

top of each bar indicates the amount of iterations terminated with a solution. As expected, a general tendency of larger neighborhoods leading to overall larger diversity is seen. Furthermore, despite a slightly smaller total amount of solutions included in the $Q_{\text {sum }}$ runs, the solutions achieved here contain a significant higher degree of diversity. As concluded in Section 5.1, the single run covering the largest difference is the second $\left(i_{2}\right)$ iteration, in which the differences are identical for the $Q_{\text {maxmin }}$ and $Q_{\text {sum }}$ approaches. The higher degree of diversity seen in the $Q_{\text {sum }}$ runs are therefore caused by the investment solutions of the remaining iterations deviating more from the optimal solutions (see Figure 14 in Appendix D). Contrarily, the remaining iterations of the $Q_{\operatorname{maxmin}}$ runs are seen to be more similar to the optimal solution. Therefore, if the goal is to find the single most deviating investment strategy, the $Q_{\text {maxmin }}$ and $Q_{\text {sum }}$ perform equally well, but if one looks for a portfolio of solutions with generally higher diversity, the $Q_{\text {sum }}$ might provide better results. As an illustrative example, Figure 15 and Figure 16 in Appendix D show the iteration-wise investment strategy of the PoMDS when the $Q_{\text {maxmin }}$ and $Q_{\text {sum }}$ are applied, respectively. The graphs cover the $P_{2014}$ with a $5 \%$ limit on the change in system costs. From these figures, both the individual development in investment strategies are seen throughout the PoMDS iterations and the differences in these developments between the $Q_{\operatorname{maxmin}}$ and $Q_{\text {sum }}$ runs.

Since the purpose of this analysis is to illustrate the properties of the PoMDS algorithm, the case study is based on a sandbox problem, wherefore the single investment strategies are less essential to analyze. Nevertheless, Figure 4 illustrates the investment strategies of each of the 180 runs. This figure gives an indication of the diversity in the different solutions in relation to total amount of invested units. Moreover it illustrates how the size of the neighborhood might influence the investment strategies found in each iteration. When the PoMDS approach is applied to a real-size energy system problem, a figure of this type is of course essential as it provides the different investment strategies to be presented for a potential decision maker. 

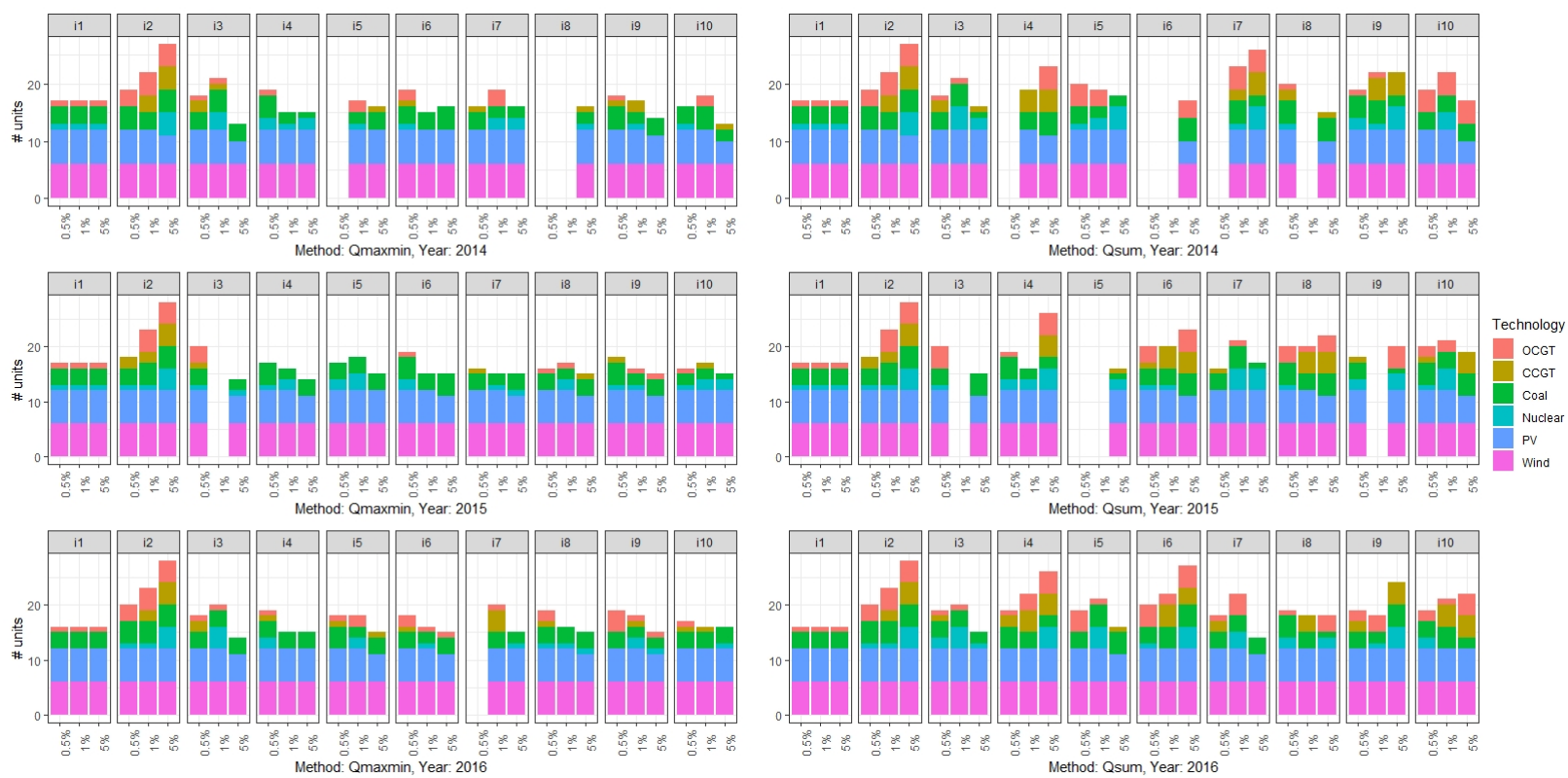

Fig. 4: Investment strategies for each iteration run for each method tested. Investment strategies equal to the zero investment are caused by the termination due to the time limit, where no solution was found.

An essential observation from Figure 4 is the investment decisions which are frequently made among the different strategies. By analyzing the occurrences of a single unit investment among all runs, an indication of the relative importance of this unit investment is achieved. Doing so for each single unit investments, a graph as seen in Figure 5 is obtained. Disregarding the no-solution
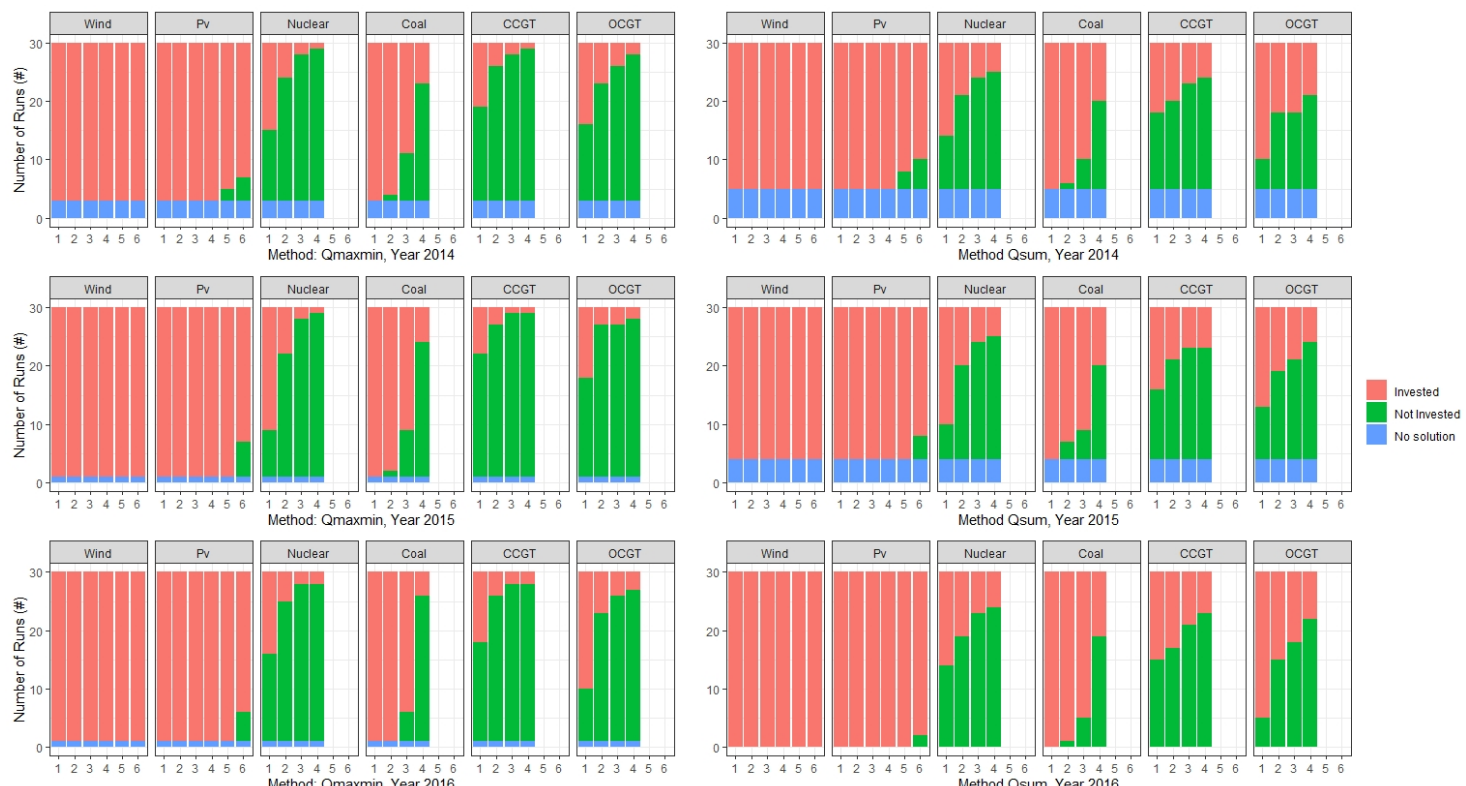

Fig. 5: The likeliness of an investment being made based on the results of the 180 runs.

iterations, it is seen that the $Q_{\operatorname{maxmin}}$ and the $Q_{\text {sum }}$ runs share the same tendency, however with generally more units being frequently used in the $Q_{\text {sum }}$ runs. We claim that, with a single unit investment being performed in a high share of the runs, it indicates that this unit investment not only is important to the investment strategy, but also likely is a more trusted investment to be part 
of the optimal non-aggregated investment strategy. Applying the terminology of [30], we refer to an investment which is activated in each iteration, as must haves $(\mathrm{MH})$. In relation to the presented case study here, each of the units related to the wind technology appear as (MHs) (see Figure 5). The same goes for the majority of the units related to the PV technology and to some degree also some of those related to the Coal technology. Further following the terminology of [30], unit investments appearing in at least one iteration are referred to as Real Choices (RC). As an extension to this definitions we introduce multiple levels of the real choices, such as weak and strong levels; $R C_{W}$ and $R C_{S}$, indicating different degree of membership to the total amount of solutions. From Figure 5 we see that, in our sandbox toy problem, every unit possible for investments is included in the set of (RCs). Therefore this problems does not have any Must Avoids (MA), defined as unit investments never performed by any run [30]. An illustration of this terminology is seen in Figure 6, highly inspired by $[30]$.

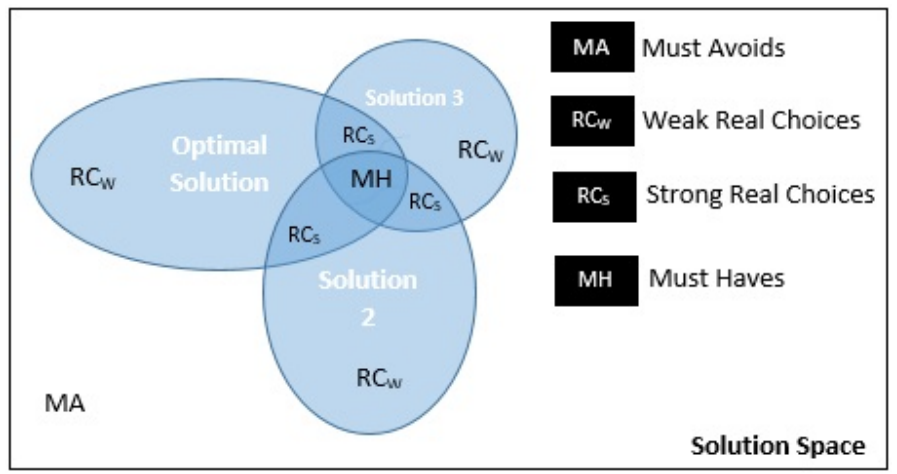

Fig. 6: Illustrative explanation of the different categorized investment decisions. The illustrative example contains a solution space with one optimal and two alternative solutions identified. Each investment is then categorized as either Must Avoids, Weak Real Choices, Strong Real Choices or Must Haves.

\subsection{Diversity in the Objective values}

In Section 5.1 we concluded that the investment strategy having highest diversity does not necessarily cause the largest change in system costs. Furthermore, also that the solution property of having highest change in system costs in the aggregated solution space not necessarily is pursued when projecting the solutions onto the non-aggregated solution space. In this section we look into these relations when the whole portfolio is considered. The percentage deviation in system costs is illustrated in Figure $7 \mathrm{a}$ for the aggregated problem and in Figure $7 \mathrm{~b}$ for the non-aggregated problem. First, we notice that, despite the conclusion of the MID solutions not having the largest change in system costs, the actual change in system costs for these solution seems to be in the large end of the scale. In general, holding the height of each bar against the amount of solutions included (number on the top of the bar) gives an indication of the degree to which the objective value limiting constraint is binding for each solution. The general picture, both in the aggregated and in the non-aggregated case, is that the larger the neighborhood, the more frequently iterations are terminated with a higher change in system costs. This indicates a generally good exploration of the full neighborhood size. However, it is also clear that the deviation in system costs generally tends to be smaller in the nonaggregated solution space. A consequence of using the aggregated solution to search for alternative solutions is therefore a limited exploration of the full non-aggregated neighborhood. Another very interesting relation between the two solution spaces is that the deviations in the non-aggregated case actually also turns up negative, indicating one of the alternative solutions being more cost effective than the assumed optimal one. This is again a consequence of the decrease in solution quality when aggregation techniques are applied [19]. However, it also strengthen the applicability of the PoMDS algorithm as this approach then counteracts the errors introduced by aggregating. Nevertheless, as already mentioned, cases in which the non-aggregated neighborhood is exceeded also occurs $(4 \%$ of the tests, equally distributed on the $Q_{\text {maxmin }}$ and $Q_{\text {sum }}$ runs) with the highest violation being $3 \%$. 

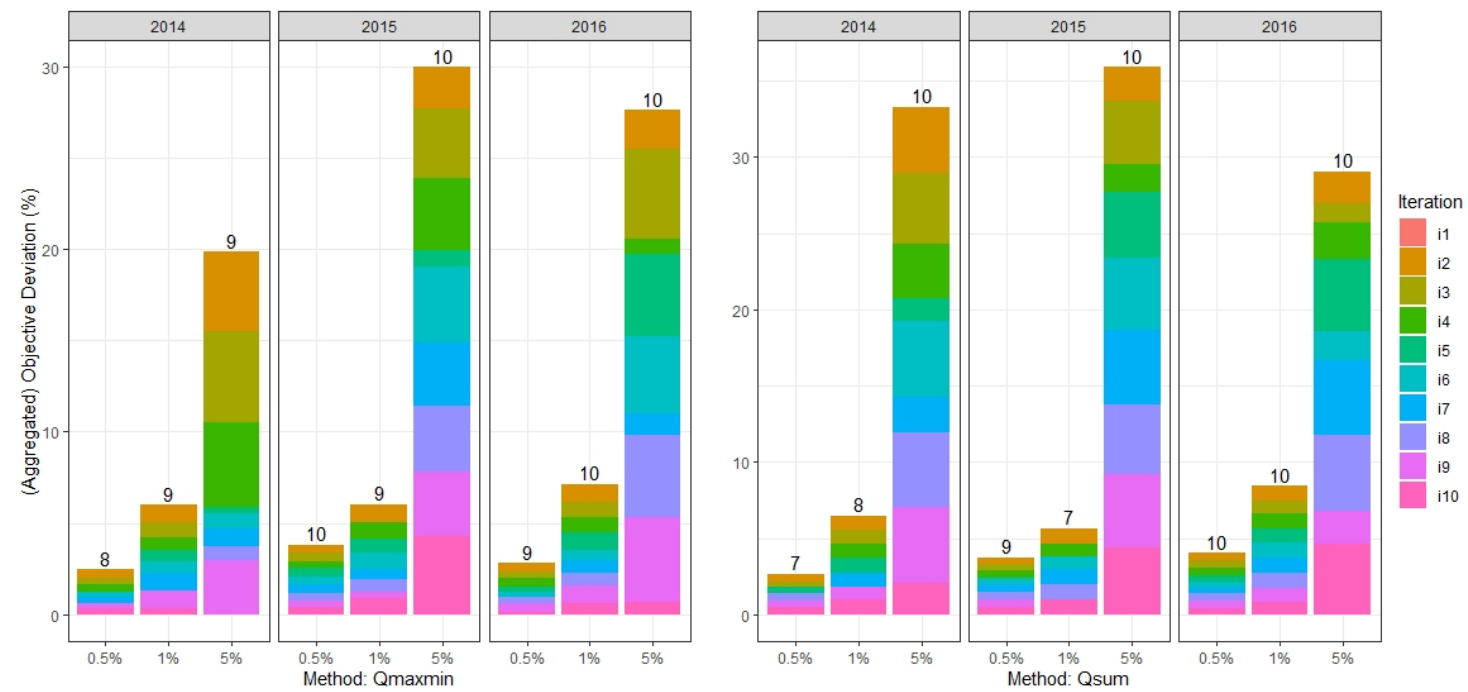

(a) Aggregated Objective
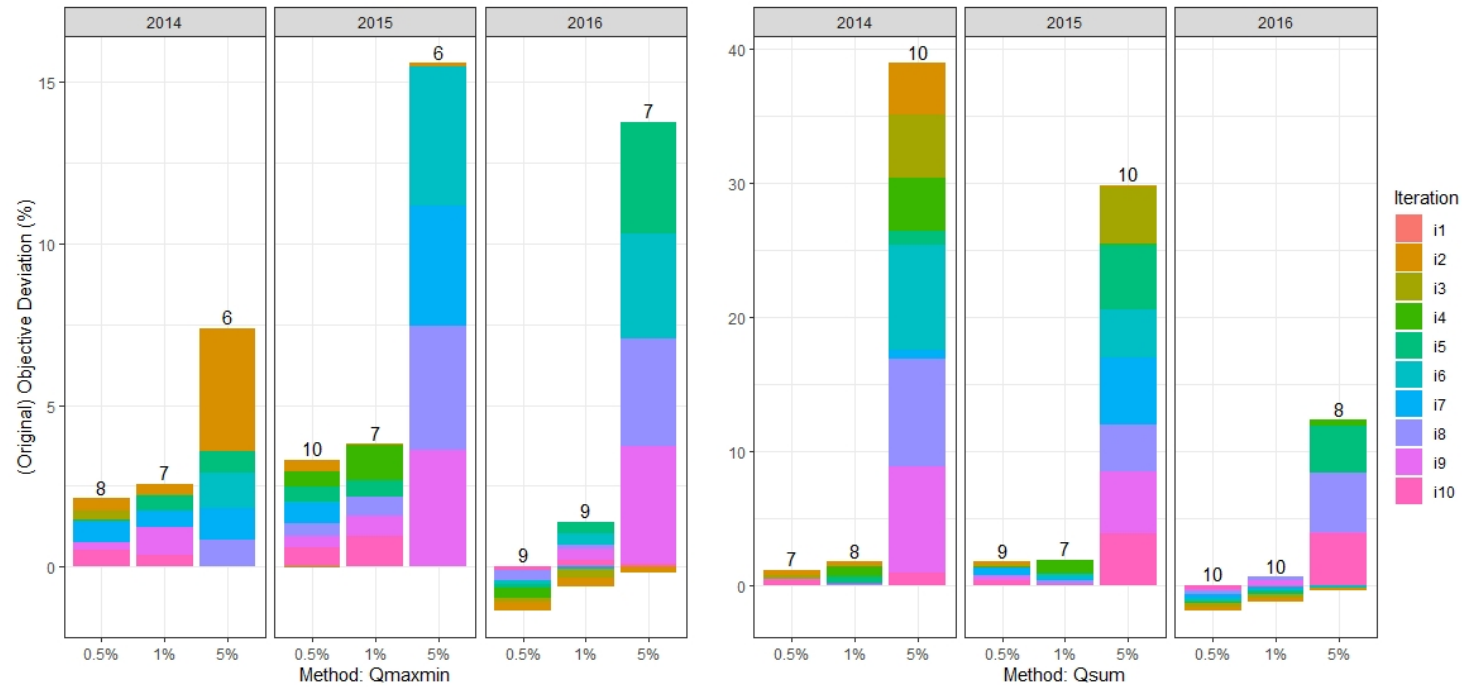

(b) Non-aggregated Objective

Fig. 7: The percentage deviation from the objective of iteration run 1 to the objective of all other iteration runs. Figure $7 \mathrm{a}$ shows the deviations observed in the aggregated problem, while Figure $7 \mathrm{~b}$ shows deviations concerning the original objective. The number on top of the bars indicates the amount of solutions covered by the bar.

\subsection{Solution Times}

One of the advantages of the PoMDS algorithm is that it provides a portfolio of investment strategies based on the aggregated problem in comparable time to a single run of the non-aggregated problem. As argued in Section 1, a lot of uncertainties are influencing the non-aggregated problem, a portfolio of strategies might bring more valuable information, despite these being based on the aggregated solution space. However, with the added layer of investment diversity maximization increasing the complexity of the problem, it is essential to analyze the solution times for verifying, under which conditions, the claim of comparable solution times of the aggregated portfolio and the single nonaggregated run, holds. For this purpose, each non-aggregated problem is solved to a $0.01 \%$ optimality gap, from which the following solutions times are obtained; $T_{P_{2014}}=20466 \mathrm{~s}, T_{P_{2015}}=16351 \mathrm{~s}$ and $T_{P_{2016}}=8114 s$. Due to the PoMDS algorithm being performed with a time limit, the choice of time limit, naturally influences the overall solution time of the portfolio. Figure 8a illustrates the iterationwise accumulated solution times for each method with a two hour limitation on the solution time 
of each iteration, while Figure $8 \mathrm{~b}$ shows the solution times when interrupted runs are disregarded. Notice that, iteration $i_{1}$ is the optimal solution run and it is succeeded in all cases, wherefore it is
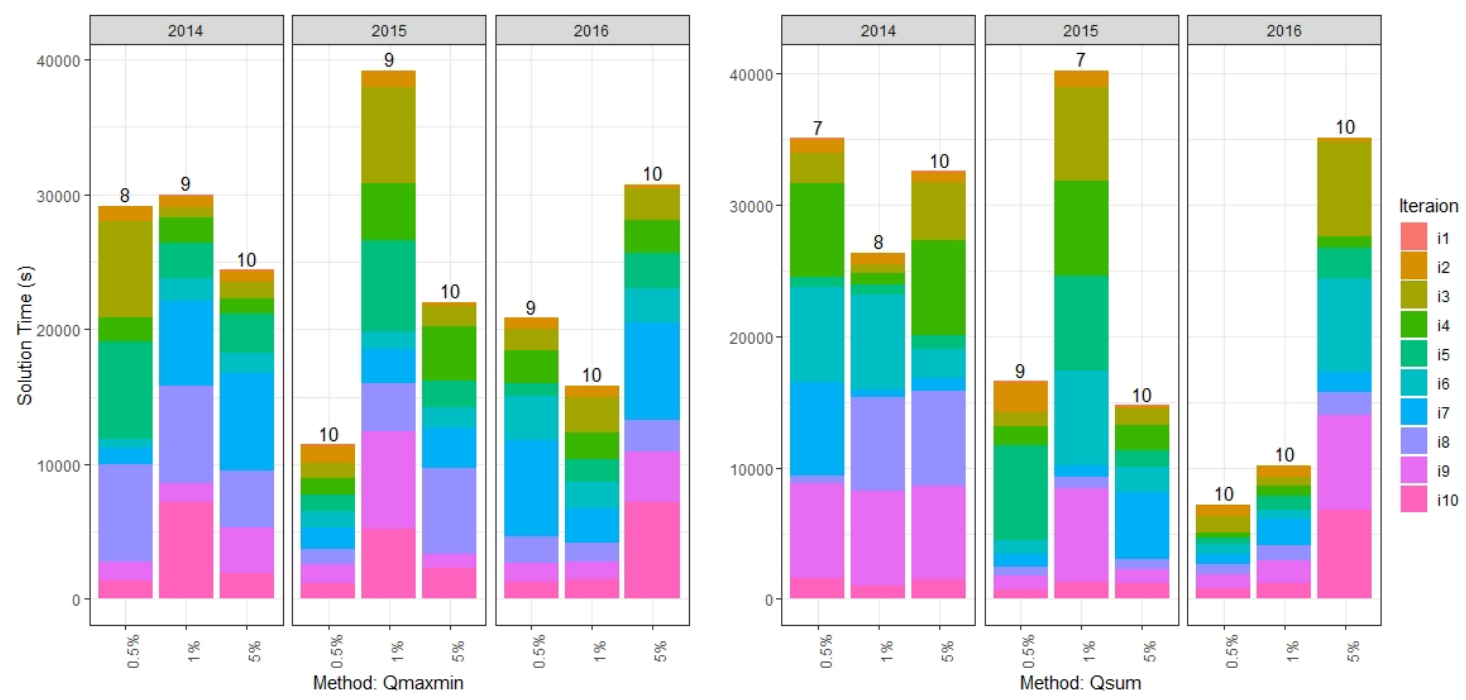

(a) Solution Times of all iteration
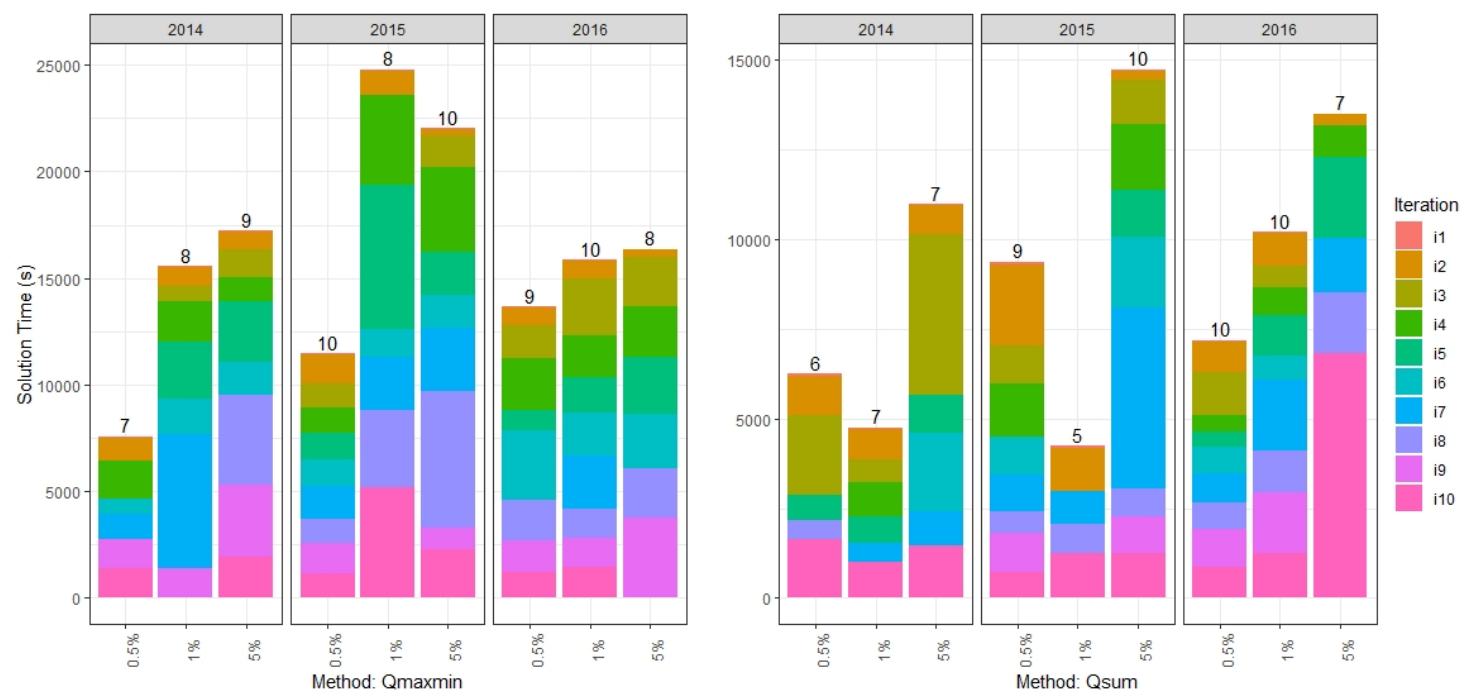

(b) Solution Times of non-interrupted iterations

Fig. 8: Solution Times of the different iteration for each method. Figure 8 a includes the 7200 s time limit for interrupted runs wherefore the number on top of each bar indicates the amount of solutions found in all iterations. Figure 8b disregards interrupted runs and the number on top of the bars therefore indicate the amount of iterations not terminated due to the time limit.

included in each stacked bar in both figures. However, due to the solution times related to these runs being significantly lower compared to the other runs, the bar is not visible in the graphs.

First, we notice that, despite a negative correlation between solution time and neighborhood sizes being observed for iteration $i_{2}$, neither of the figures seems to indicate any such relation. Contrarily, a slight tendency of larger neighborhoods more frequently causing longer solution times per iteration are seen from Figure 8b. Looking into the total solution time (Figure 8a) it is seen that generally similar solution times are observed for the $Q_{\operatorname{maxmin}}$ and the $Q_{\text {sum }}$ runs. In both cases the maximum completion time of ten iterations appears for problem $P_{2015}$ associated to a $1 \%$ neighborhood, and the time is in both cases around 40000s. The quality of the two iteration is, however, higher for the $Q_{\operatorname{maxmin}}$ method. On the other hand, higher quality and much lower solution times are seen for the $Q_{\text {sum }}$ runs when the $P_{2016}$ problem is considered. In fact, using a $0.5 \%$ neighborhood in the 
$Q_{\text {sum }}$ method provides a portfolio of ten different solutions without exceeding the solution time of the non-aggregated problem. With a portfolio size of ten solutions and a time limit of two hours, this property is not seen for each tested problem.

\subsubsection{Tuning the time limit parameter}

Figure 9 illustrates the share of iterations which are completed within the solution times of the nonaggregated problems. From this it is seen that in 11 out of the 18 tests, it is possible to complete at least eight iterations, which, in best case, provides eight different solutions. In all but one test, five iterations are possible to complete and in four tests all ten iterations are completed. On average, seven iterations are completed in the $Q_{\text {maxmin }}$ runs while this is increased to eight when the $Q_{\text {sum }}$ runs are considered. Now, to study the effect of the time limit, an estimation of similar test runs with

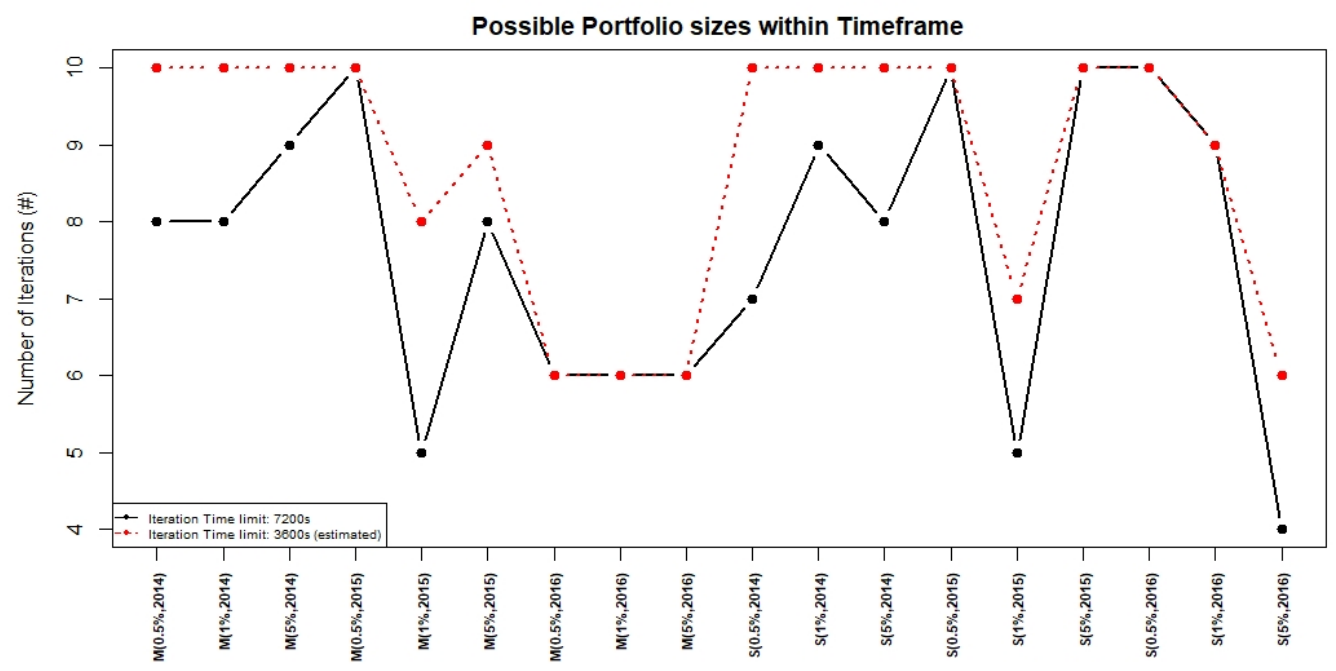

Fig. 9: Illustration of the share of iterations possible to complete within the time it takes to solve the non-aggregated problem with a $0.01 \%$ optimality gap. Also an estimation of the affect of decreasing the time limit is shown.

a one hour limitation are performed and showed by the dotted line in Figure 9. We refer to this as an estimation since no new test runs are performed, but we simply adjust the time limit in the already achieved run times. Consequently, this adds 11 more iterations to the interruption category, wherefore the quality of these, and the quality of the already interrupted iterations, potentially decrease (if runs with a one hour limitation actually were performed). Nevertheless, by changing the time limit we now see ten iterations being completed within the timeframes in ten out of the 18 tests. The average completion is increased by one for both approaches meaning that an average $Q_{\text {maxmin }}$ run completes eight iterations while an average $Q_{\text {sum }}$ run now completes nine iterations. Of course this should be considered in relation to the potential quality decrease, wherefore these results only are an indication of the potential for tuning the parameter values of the PoMDS algorithm.

\subsection{Results of the NOS approach}

To highlight the benefits of the PoMDS algorithm, we compare the above results to similar results achieved from the NOS approach. The comparison is made for each of the neighborhood sizes selected for the PoMDS. We therefore study, in which iteration the neighborhood specific investment differences achieved in the PoMDS, are found by the NOS. In each case we state the iteration, the solution time and the change in system costs in aggregated and non-aggregated solution spaces obtained for this iteration. These results are seen in Table 3. Since the NOS algorithm was terminated after 48 hours, a solution matching the PoMDS solution of the $5 \%$ neighborhood was not obtained. Consequently, the stated numbers for this neighborhood indicate how far the NOS algorithm came 
Table 3: Overview and specifics of the NOS iteration which provide similar diversity solutions as obtained for each neighborhood in the PoMDS algorithm. The sign '>' indicates that the actual numbers from the NOS algorithm would be higher than the stated, if the solution was to match the one achieved in the PoMDS algorithm. However, due to the overall termination time, the stated numbers indicate how far the NOS algorithm came.

\begin{tabular}{|c|c|c|c|c|c|}
\hline & \multicolumn{3}{|c|}{ Max. Investment Diff. } & \multicolumn{2}{|c|}{ Maximum change in System Cost } \\
\hline Name & It. & Value & Time & Value (Agg.) & Value (non-Agg.) \\
\hline$N_{O S} S_{2014}$ (matching $0.5 \%$ neighborhood) & 26 & 4 & 2942 & 0.42 & 0.27 \\
\hline$N O S_{2014}$ (matching $1 \%$ neighborhood) & 132 & 7 & 129645 & 0.91 & 0.05 \\
\hline$N_{O} S_{2014}$ (matching 5\% neighborhood) & $>149$ & $>7$ & $>169368$ & 0.95 & 0.47 \\
\hline 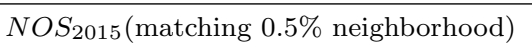 & 15 & 3 & 3111 & 0.38 & 0.33 \\
\hline$N O S_{2015}$ (matching $1 \%$ neighborhood) & 137 & 6 & 74347 & 0.99 & 0.07 \\
\hline$N O S_{2015}$ (matching $5 \%$ neighborhood) & $>193$ & $>6$ & $>168163$ & 1.18 & 0.4 \\
\hline$N O S_{2016}$ (matching $0.5 \%$ neighborhood) & 28 & 4 & 2391 & 0.48 & -0.28 \\
\hline$N O S_{2016}$ (matching $1 \%$ neighborhood) & 124 & 7 & 110793 & 0.98 & -0.25 \\
\hline$N O S_{2016}$ (matching $5 \%$ neighborhood) & $>144$ & $>7$ & $>165492$ & 1.06 & 0.37 \\
\hline
\end{tabular}

and the sign ' $>$ ' indicates that the actual number would be higher if more time was given. Comparing the results in Table 2 ( $0.5 \%$ neighborhood) to the corresponding results in Table 3 , we see that the NOS algorithm requires 15-28 solutions before reaching the same amount of investment differences as the PoMDS solution. Consequently, the solution time is 2-2.8 times higher than the corresponding solution times of the PoMDS (with highly similar change in system costs both in the aggregated and in the non-aggregated space). Going to the $1 \%$ neighborhood solutions it is now seen that the NOS require 124-137 iterations to obtain the same amount of investment differences as was found by the PoMDS in the second iteration. This means that the NOS algorithm would spend 21-36 hours to find the solution which by the PoMDS is found in 831-1201s. However, the solution found by the PoMDS are associated to the slightly higher changes in system costs especially in the non-aggregated solution space. For the $5 \%$ neighborhood, a direct comparison cannot be made since the NOS algorithm is not able to find a portfolio of sufficient diverse solution within the $48 \mathrm{~h}$ time limit. However, what can be concluded is that the NOS algorithm within 48 hours is not able to find a solution which differentiates itself to the optimal one with more than 25\% whereas the PoMDS finds solutions diverging with up to $43 \%$ in $300 \mathrm{~s}$. This clearly illustrates the advantage of the proposed PoMDS algorithm and we argue that this advantage is strengthen as we search for more diverse solutions. To verify this, we study the evolution of solution time, change in system cost and investment differences throughout the iterations which is illustrated in Figure 10.

Figure 10a shows that the diversity in investments increases as more iterations are completed, but also that the solution time seems to increase a lot as later iterations are reached. For the change in system costs, Figure 10b shows a nice logarithmic increase for each problem in the aggregated solution space while this trend is far from projected to the non-aggregated space where instead shifting high and low changes are seen. However, the changes do have an overall increase in the sense that as later iterations are reached the high change in system costs are obtained. Notice that, again the change in system costs in the non-aggregated space tends to be negative for the $P_{2016}$. This means that, the system costs found in the alternative solutions are more optimal than the assumed optimal solution, which is a consequence of the small errors introduced by aggregating. As more iterations are completed, it seems that the changes in system costs become more often positive and that the negative changes decrease in size. To study the impact in solution time of obtaining larger changes in system costs, a direct comparison is seen in Figure 11. Here, the change in system costs of the aggregated system are shown together with the accumulated solution time when the NOS is applied to each of the three problems $P_{2014}, P_{2015}$ and $P_{2016}$. It is here even more clearly seen that the initial $0.8 \%$ change in system costs actually is obtained fairly cheaply with respect to solution time ( 5 hours) and that it is the changes $>0.8 \%$ that takes a lot of time to obtain. From Figure 8 it was seen that the PoMDS algorithm found portfolios of approximately ten solutions in a little more than 7000-40000s corresponding to 2-11 hours. Therefore, these time limits are illustrated in Figure 11 wherefrom it is seen that the solutions achieved within a two hour limit differentiates with $0.5 \%-0.65 \%$ and within an 11 hour time frame a change of $0.7 \%-0.88 \%$ is achieved. The portfolio being found within two hours 

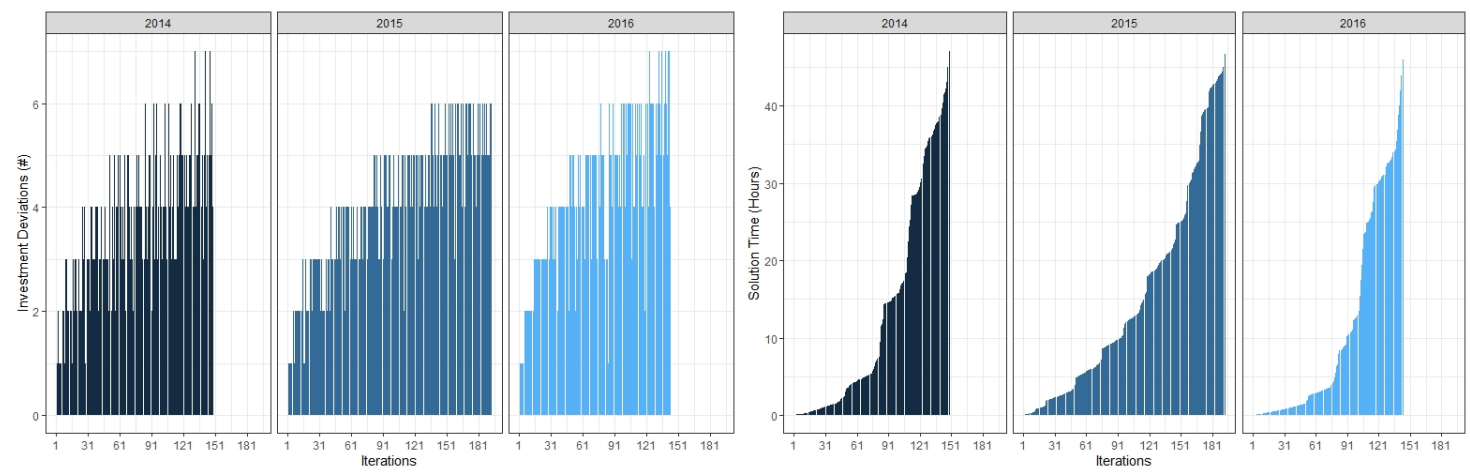

(a) Illustration of the difference in investment costs between the optimal solution and the solution found in each iteration in the NOS algorithm. Additionally, the evolution in solution time throughout the iterations are seen.
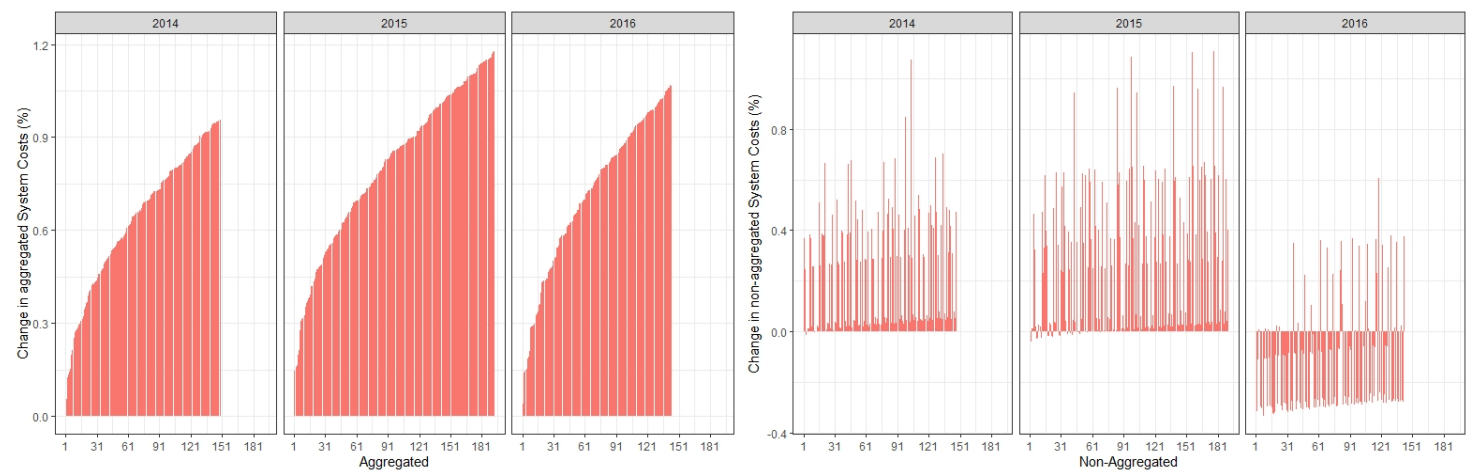

(b) Illustration of how the difference in system costs evolves throughout the iterations both within the aggregated and within the non-aggregated solution space.

Fig. 10: Illustration of how the difference in investments, change in system costs and solution time evolve through the iterations of the NOS algorithm. In Figure 10a the accumulated solution time and the difference in investments are seen. Figure 10b shows the change in system costs both within the aggregated and the non-aggregated solution space.

with the PoMDS is associated to a $0.5 \%$ wherefore the solutions are comparable to the ones achieved with the NOS algorithm both according to change in system costs and to deviations in investments. However, as the 11 hour time limit is the overall highest for any portfolio obtained from the PoMDS algorithm, the maximum difference in investments is in this case 12 associated to a $5 \%$ change in system costs. With the NOS algorithm, the 11 hour time limit means that no solution differentiating with more than 5 (6 in $\left.P_{2016}\right)$ units and $0.88 \%$ (0.7\% in $\left.P_{2016}\right)$ system costs is found. 


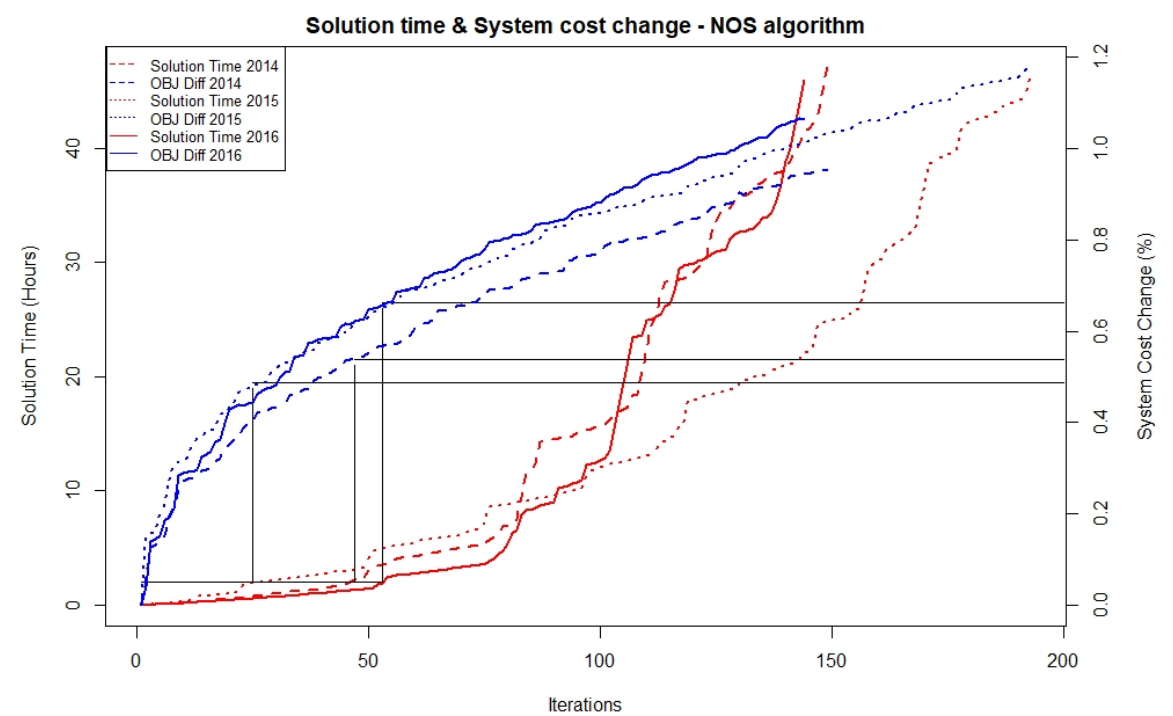

(a) Relation between solution time and aggregated system costs change with $2 \mathrm{~h}$ indicated.

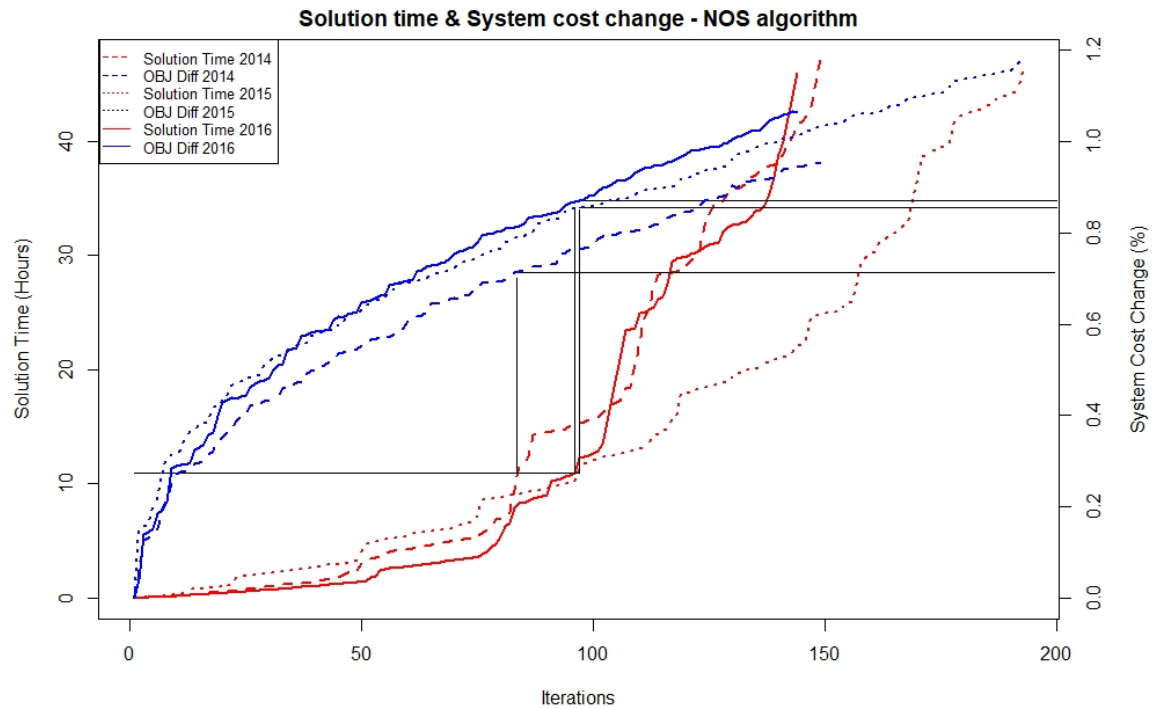

(b) Relation between solution time and aggregated system costs change with $11 \mathrm{~h}$ indicated.

Fig. 11: Illustration of the iteration-wise trade-off between solution time and change in system costs for the NOS algorithm applied to the aggregated solution space. 


\section{Conclusions}

In this paper, we suggested the Portfolio of Maximized Diversity Solutions (PoMDS) algorithm to explore the near optimal solution space such that the diversity in investment strategies are maximized. This approach challenges the idea of just searching for one optimal solution and hereby we introduce a new way of accounting for errors introduced by simplifications. A model of an energy system typically constitutes a simplification of the real world, wherefore near optimal solutions might be as good as the optimal one. Providing a decision maker with a diversity of solutions therefore provides a stronger picture. Furthermore, such a set of solutions also indicate the relative importance of each investment decisions.

The value and applicability of the PoMDS algorithm was illustrated through a case study covering different small capacity expansion problems.

Two alternative objectives for maximizing the investment differences were proposed. Either the summed differences between alternative and optimal solutions were maximized $\left(Q_{\text {sum }}\right)$, or the minimum distance between alternative solutions and optimal solution was maximized $\left(Q_{\operatorname{maxmin}}\right)$. Results showed that the $Q_{\text {sum }}$ provided higher diversity among the alternative solutions compared to the $Q_{\text {maxmin }}$ objective and that the solution quality in the non-aggregated solution space was higher with the $Q_{\text {sum }}$. However a higher share of the iteration related to the $Q_{\text {sum }}$ runs were terminated by the time limit. The recommendations for the two alternative methods therefore depend on the overall diversity needs.

Furthermore, it was seen that by allowing a $5 \%$ change in system costs the PoMDS algorithm found an investment strategy deviating with more than $40 \%$ from the optimal one within $5-14$ min. Comparing this to the so far best known Near Optimal Solution (NOS) algorithm from the literature $[31,30]$, a solution with that level of diversity was not possible to find within 48 hours. Furthermore, even when increasing the near optimal solution space the maximized diversity solution is found in the first iteration by the PoMDS algorithm whereas the NOS algorithm requires more iterations and for both algorithms the solution time increases with the amount of iterations performed.

Consequently, for small gap values the advantages of the proposed method above the one known from the literature is limited, but the advantages grow rapidly as the gap values are increased, which in our case study appeared already at a maximum change in system costs of $0.7 \%$.

Lastly, the case study clearly illustrated how the PoMDS approach provided relative importance of the different investments. From the resulting portfolio of solutions, each investment was categorized according to their appearance. We here encourage further research to study whether this potentially could be used to identify optimal investments of the non-aggregated problem. One might be able to fix certain investments in the non-aggregated space based on the categorization of the aggregated space, and then search for alternative solutions in the non-aggregated space using the PoMDS approach on the original data.

\section{Financial and Ethical disclosures}

Funding: This study was funded by the FUTUREGAS research project. Conflict of Interest: The authors declare that they have no conflict of interest.

\section{References}

1. Tomas Kåberger. Progress of renewable electricity replacing fossil fuels. Global Energy Interconnection, 1:48-52, 2018.

2. Matthew D.Leonard, Efstathios E.Michaelides, and Dimitrios N.Michaelidesr. Energy storage needs for the substitution of fossil fuel power plants with renewables. Renewable Energy, 145:951-962, 2019.

3. Gonzalo E.Alvarez, Marian G.Marcovecchio, and Pío A.Aguirre. Optimization of the integration among traditional fossil fuels, clean energies, renewable sources, and energy storages: An milp model for the coupled electric power, hydraulic, and natural gas systems. Computers \&6 Industrial Engineering, 139:106-141, 2020.

4. Cedric De Jonghe, Erik Delarue, Ronnie Belmans, and William D'haeseleer. Determining optimal electricity technology mix with high level of wind power penetration. Energy Systems, 88:2231-2238, 2011.

5. Bryan Palmintier and Mort Webster. Impact of unit commitment constraints on generation expansion planning with renewables. IEEE, Power and Energy Society General Meeting, 2011.

6. Daniel S. Kirschen, Juan Ma, Vera Silva, and Régine Belhomme. Optimizing the flexibility of a portfolio of generating plants to deal with wind generation. Ieee Power and Energy Society General Meeting, 2011.

7. Vishwamitra Oree, Sayed Z. Sayed Hassen, and Peter J. Fleming. Generation expansion planning optimisation with renewable energy integration: A review. Renewable and Sustainable Energy Reviews, 69:1369-1394, 2017. 
8. Nikolaos E. Koltsaklis and Athanasios S. Dagoumas. State-of-the-art generation expansion planning: A review. Applied Energy, 230:563-589, 2018.

9. Hans-Kristian Ringkjøb, Peter M.Haugan, and Ida MarieSolbrekke. A review of modelling tools for energy and electricity systems with large shares of variable renewables. Renewable and Sustainable Energy Reviews, 96:440-459, 2018.

10. Anna Schwele, Jalal Kazempour, and Pierre Pinson. Do unit commitment constraints affect generation expansion planning? a scalable stochastic model. Energy Systems, pages 1-36, 2019.

11. Venkat Krishnan and Wesley Cole. Methods for multi-objective investment and operating optimization of complex energy systems. Ieee Power and Energy Society General Meeting, page 7741996, 2016.

12. Karl Kiên Cao, Johannes Metzdorf, and Sinan Birbalta. Incorporating power transmission bottlenecks into aggregated energy system models. Sustainability (Switzerland), 10:1916, 2018.

13. Fernando J. de Sisternes and Mort D Webster. Optimal selection of sample weeks for approximating the net load in generation planning problems. Massachusetts Institute of Technology. Engineering Systems Division, 2013.

14. Holger Teichgraeber and Adam R. Brandt. Clustering methods to find representative periods for the optimization of energy systems : An initial framework and comparison. Applied Energy, 239:1283-1293, 2019.

15. James H. Merrick. On representation of temporal variability in electricity capacity planning models. Energy Economics, 59(19):261-274, 2016.

16. Francisco D. Munoz and Andrew D. Mills. Endogenous assessment of the capacity value of solar pv in generation investment planning studies. Transactions on Sustainable Energy, 6(4):1574-1585, 2015.

17. Kris Poncelet, Hanspeter Hoschle, Erik Delarue, Ana Virag, and William D'haeseleer. Selecting representative days for capturing the implications of integrating intermittent renewables in generation expansion problems. IEEE Transactions on Power Systems, PP(99):1-1, 2016.

18. Henrik C. Bylling, Salvador Pineda, and Trine K. Boomsma. The impact of short-term variability and uncertainty on long-term power planning problems. Annals of Operational Research, 239:1-27, 2017.

19. Stefanie Buchholz, Mette Gamst, and David Pisinger. A comparative study of aggregation techniques in relation to capacity expansion energy system modeling. TOP 2019, Issue 3, 2019.

20. Majid Taghavi and Kai Huang. Stochastic capacity expansion with multiple sources of capacity. Operations Research Letters, 14:263-267, 2014

21. Victor Hinojosa, Esteban Gil, and Ignacio Calle. A stochastic generation capacity expansion planning methodology using linear distribution factors and hourly load modeling. 2018 International Conference on Probabilistic Methods Applied To Power Systems, Pmaps 2018 - Proceedings, 88:8440244, 2018.

22. Mette Gamst. Sifre: Simulation of flexible and renewable energy sources.

23. Hans Ravn. The balmorel model structure.

24. T. S. Chung, Y. Z Li, and Z. Y Wang. Optimal generation expansion planning via improved genetic algorithm approach. International Journal of Electrical Power and Energy System, 26:655-659, 2004.

25. Adelino J.C Pereira and João Tomé Saraiva. Generation expansion planning (gep) - a long-term approach using system dynamics and genetic algorithms (gas). International Journal of Electrical Power and Energy System, 65:5180-5199, 2011.

26. S. Safari, M. M. Ardehali, and M. J. Siriz. Particle swarm optimization based fuzzy logic controller for autonomous green power energy system with hydrogen storage. Energy Conversion and Management, 36:41-49, 2013.

27. Bryan S. Palmintier. Incorporating operational flexibility into electric generation planning : impacts and methods for system design and policy analysis. Massachusetts Institute of Technology, 2013.

28. Clara F.Heuberger, Edward S.Rubind, Iain Staffell, Nilay Shah, and Niall Mac Dowell. Power capacity expansion planning considering endogenous technology cost learning. Applied Energy, 204:831-845, 2017.

29. Berit Müller, Francesco Gardumi, and Ludwig Hülk. Comprehensive representation of models for energy system analyses: Insights from the energy modelling platform for europe (emp-e) 2017. Energy Strategy Reviews, 21:82$87,2018$.

30. Philip Voll, Mark Jennings, Maike Hennen, Nilay Shah, and André Bardow. The optimum is not enough: A near-optimal solution paradigm for energy systems synthesis. Energy, 82:446-456, 2015.

31. Samira Fazlollahi, Pierre Mandel, Gwenaelle Becker, and Francois Maréchal. Methods for multi-objective investment and operating optimization of complex energy systems. Energy, 45:12-22, 2012.

32. Pei Liu, Efstratios N. Pistikopoulos, and Zheng Li. An energy systems engineering approach to the optimal design of energy systems in commercial buildings. Energy Policy, 38:4224-4231, 2010.

33. H. Gebreslassie Berhane, Guillén Gosálbez Gonzalo, Jiménez Laureano, and Boer Dieter. Design of environmentally conscious absorption cooling systems via multi-objective optimization and life cycle assessment. Applied Energy, 86:1712-1722, 2009.

34. Matteo Fischetti and Michele Monaci. Proximity search for 0-1 mixed-integer convex programming. Journal of Heuristics, 20:709-731, 2014.

35. Olubayo M. Babatunde, Josiah L. Munda, and Yskandar Hamam. A comprehensive state-of-the-art survey on power generation expansion planning with intermittent renewable energy source and energy storage. Energy Research, 2019.

36. Energinet.dk. Markedsdata.

37. Fernando J. de Sisternes, Mort D. Webster, and Ignacio J. Pérez-Arriaga. The impact of bidding rules on electricity markets with intermittens renewables. IEEE Transactions on Power Systems, 30(3):1603-1613, 2015.

38. Falko Ueckerdt, Robert Brecha, and Gunnar Luderer. Analyzing major challenges of wind and solar variability in power systems. Renewable Energy, 81(1):1-10, 2015.

39. Kris Poncelet, Erik Delarue, Daan Six, Jan Dueinck, and William D'haeseleer. Impact of the level of temporal and operational detail in energy-system planning models. Applied Energy, 162(58):631-643, 2015.

40. IRENA (2017). Planning for the renewable future: Long-term modelling and tools to expand variable renewable power in emerging economies. International Renewable Energy Agency, Abu Dhabit, 2017. 


\section{Nomenclature}

$\epsilon \quad$ Maximum change in System Cots (Neighborhood Size)

Agg. Aggregated

ATQ Aggregation Technique

CCGT Closed Cycle Gas Turbine

$C E P$ Capacity Expansion Problems

$D S \quad$ Data Series

$D S_{a g g}$ Aggregated Data Series

ES Exhaustive Search

GEP Generation Expansion Problems

$I R_{x} \quad$ Irregular Run, type $x$

$M A \quad$ Much Avoids

$M D S$ Maximized Diversity Solution

$M H \quad$ Must Haves

MID Maximized Investment Different solution

non - Agg. Non Aggregated

NOS Near Optimal Solution

OCBT Open Cycle Gas Turbine

$P_{x} \quad$ Capacity expansion problem based on data series $x$

PoMDS Portfolio of Maximized Diversity Solutions

$P V \quad$ Photovoltaics

$Q_{\text {maxmin }}$ Problem where the objective maximizes the minimum distance to the previous solutions

$Q_{\text {sum }}$ Problem where the objective maximizes the sum of distances to all previous solutions

$R C \quad$ Real Choices

$R C_{S} \quad$ Strong Real Choices

$R C_{W}$ Weak Real Choices

$R L D C$ Residual Load Duration Curve

$T_{\max }$ Time limit for each iteration

$V R E \quad$ Variable Renewable Energy

\section{A Mathematical Formulation}

Equation (6)-(19) cover the mathematical formulation of the capacity expansion model, where expansion costs are minimized subject to technical and operational constraints. A detailed description of the constraints is provided right after the mathematical model. Sets, parameters and variables are listed in Table 4 while Table 5 provides an overview of the specific values applied in our case study. Notice that, for simplicity reasons, it is assumed that the variable costs are equal for all hours.

$$
\begin{array}{cll}
\text { Minimize } \sum_{i \in I}\left(C_{i}^{I N V}+C_{i}^{F O M}\right) y_{i}+\sum_{i \in I} \sum_{j \in J} & \left(C_{i}^{V A R}+C_{i}^{V O M}+P_{i}^{F U E L} H R_{i}\right) x_{i j}+C_{i}^{S T U P} z_{i j} \\
\text { Subject to } & & \forall j \in J \\
\sum_{i \in I} x_{i j} \geq D_{j} & \forall i \in I, \forall j \in J \\
x_{i j} \leq \bar{P}_{i} y_{i} & \forall i \in I^{W}, \forall j \in J \\
x_{i j} \leq \bar{P}_{i} C F_{j}^{W I N D} & \forall i \in I^{S}, \forall j \in J \\
x_{i j} \leq \bar{P}_{i} C F_{j}^{S O L A R} & \forall i \in I^{T}, \forall j \in J \backslash\{1\} \\
u_{i j}-u_{i j-1}=z_{i j}-v_{i, j} & \forall i \in I^{T}, \forall j \in J \\
w_{i j}=x_{i j}-u_{i j} \underline{P}_{i} & \forall i \in I^{T}, \forall j \in J \\
w_{i j} \leq u_{i j}\left(\bar{P}_{i}-\underline{P}_{i}\right) & \forall i \in I^{T}, \forall j \in J \backslash\{1\} \\
w_{i j}-w_{i j-1} \leq \bar{R}_{i}^{U} & \forall i \in I^{T}, \forall j \in J \backslash\{1\} \\
w_{i j-1}-w_{i j} \leq \bar{R}_{i}^{D} & \forall i \in I^{T}, \forall j \in J \\
u_{i j} \geq \sum_{j^{\prime}>j-\bar{M}_{i}^{U}}^{j} z_{i j^{\prime}} & \forall i \in I^{T}, \forall j \in J \\
1-u_{i j} \geq \sum_{j^{\prime}>j-\bar{M}_{i}^{D}}^{j} v_{i j^{\prime}} & \forall i \in I, \forall j \in J \\
y_{i}, u_{i j}, v_{i j}, z_{i j} \in\{0,1\} & \forall i \in I, \forall j \in J \\
x_{i j}, w_{i j} \geq 0 &
\end{array}
$$


Table 4: Sets, Parameters and Variables of the capacity expansion model with unit commitment constraints

\begin{tabular}{|c|c|c|}
\hline Set name & Description & Alias \\
\hline $\begin{array}{c}J \\
I \\
I^{W} \\
I^{S} \\
I^{T}\end{array}$ & $\begin{array}{l}\text { Time periods of the analyzed time series } \\
\text { Units, which potentially are built } \\
\text { Units of wind type } \\
\text { Units of solar type } \\
\text { Units of thermal type (Nuclear, coal, CCGTs and OCGTs) }\end{array}$ & $\begin{array}{l}\left\{j, j^{\prime}\right\} \\
\quad i \\
I^{W} \subset I \\
I^{S} \subset I \\
I^{T} \subset I\end{array}$ \\
\hline Parameter & Description & Unit \\
\hline $\begin{array}{c}C_{i}^{I N V} \\
C_{i}^{F O M} \\
C_{i}^{V A R} \\
C_{i}^{V O M} \\
C_{i}^{S T U P} \\
P_{i}^{F U E L} \\
H R_{i} \\
D_{j} \\
\bar{R}_{i}^{U} \\
\bar{R}_{i}^{D} \\
\bar{P}_{i} \\
\underline{P}_{i} \\
\bar{M}_{i}^{U} \\
\bar{M}_{i}^{D} \\
C F_{j}^{W I N D} \\
C F_{j}^{S O L A R}\end{array}$ & $\begin{array}{l}\text { Investment costs of unit } i \\
\text { Fixed Operation \& Management costs of unit } i \\
\text { Variable costs of unit } i \\
\text { Variable Operation \& Management costs of unit } i \\
\text { Start-up costs of unit } i \\
\text { Fuel Price of unit } i \\
\text { Heatrate of unit } i \\
\text { Demand for time period } j \\
\text { Upper Ramping limit of unit } i \text { (Maximum hour-by-hour increase in generation) } \\
\text { Lower Ramping limit of unit } i \text { (Maximum hour-by-hour decrease in generation) } \\
\text { Maximum power generation capacity of unit } i \\
\text { Minimum power generation capacity of unit } i \\
\text { Minimum amount of hours unit } i \text { needs to be online after start-up } \\
\text { Minimum amount of hours unit } i \text { needs to be offline after shut-down } \\
\text { Capacity factor of wind units in hour } j \text { (Availability of wind) } \\
\text { Capacity factor of solar units in hour } j \text { (Availability of solar) }\end{array}$ & $\begin{array}{c}\text { [\$/year] } \\
{[\$ / \text { year }]} \\
{[\$ / \mathrm{MWh}]} \\
{[\$ / \mathrm{MWh}]} \\
{[\$]} \\
{[\$ / \mathrm{MBTU}]} \\
{[\mathrm{MBTU} / \mathrm{MWh}]} \\
{[\mathrm{MWh}]} \\
{[\mathrm{MWh} / \mathrm{h}]} \\
{[\mathrm{MWh} / \mathrm{h}]} \\
{[\mathrm{MW}]} \\
{[\mathrm{MW}]} \\
{[-]} \\
{[-]} \\
{[-]} \\
{[-]}\end{array}$ \\
\hline Variable & Description & Domain \\
\hline $\begin{array}{c}y_{i} \\
u_{i j} \\
z_{i j} \\
v_{i j} \\
x_{i j} \\
w_{i j}\end{array}$ & $\begin{array}{l}\text { Building decision for unit } i \text {, if } 1 \text { the unit is built } \\
\text { Commitment state, if } 1 \text { unit } i \text { is online in time } j \\
\text { Start-up decision, if } 1 \text { unit } i \text { is started up in time } j \\
\text { Shut-down decision, if } 1 \text { unit } i \text { is shut down in time } j \\
\text { Power output decision, amount of energy generated by unit } i \text { in time } j \\
\text { Power generation over minimum capacity of unit } i \text { in time } j\end{array}$ & $\begin{aligned} & \{0,1\} \\
& \{0,1\} \\
& \{0,1\} \\
\mathbb{R}_{+} & (\text {or }\{0,1\}) \\
& \mathbb{R}_{+} \\
& \mathbb{R}_{+}\end{aligned}$ \\
\hline
\end{tabular}

The objective function (6) minimizes fixed and variable costs of investments and operations. The fixed costs $C_{i}^{F O M}$ cover investment costs and fixed O\&M costs, while the variable costs $C_{i j}^{V O M}$ consist of fuel costs, variable O\&M costs and variable operational costs. Constraint (7) ensures energy balance while constraints (8)-(10) handle the capacities. From these it is seen that curtailment is allowed at no extra cost. Constraints (11)-(17) represent the unit commitment, meaning that these account for the commitment state, updating of shut-down and start-ups, ramping restrictions and minimum up- and down times. Constraint (12) defines the auxiliary variable $w_{i j}$ as the power generated above the minimum level of the unit. In (18)-(19) the domain of the variables are defined. Note, that constraints (8) and (12) implicitly secure zero commitment state for non-built units.

Table 5: Technological parameter values assumed for the case study of this paper. We assume $\bar{R}_{i}^{U}=$ $\bar{R}_{i}^{D}$ wherefore only $\bar{R}_{i}^{U}$ is listed. The unit column refers to the amount of candidate units available for investments. The values have been adjusted such that a wide pallet of technologies are being selected in the original solution. Also, the cost of VRE technologies are kept low to make these preferable to the system in order to analyze a system highly reliable on VRE.

\begin{tabular}{|c|c|c|c|c|c|c|c|c|c|c|c|c|c|}
\hline & $\begin{array}{c}\text { Units } \\
(\#)\end{array}$ & $\begin{array}{c}C_{i}^{I N V} \\
(\$ / \text { year })\end{array}$ & $\begin{array}{c}C_{i}^{F O M} \\
(\$ / \text { year })\end{array}$ & $\begin{array}{c}C_{i j}^{V O M} \\
(\$ / \mathbf{M W h})\end{array}$ & $\begin{array}{c}P_{i}^{F U E L} \\
(\$ / \mathrm{MBTU})\end{array}$ & $\begin{array}{c}H R_{i} \\
\text { (MBTU/MWh) }\end{array}$ & $\begin{array}{c}C_{i j}^{V A R} \\
\text { (\$/MWh) }\end{array}$ & $\begin{array}{c}C_{i j}^{S T U P} \\
(\$)\end{array}$ & $\begin{array}{l}\bar{M}_{i}^{U} \\
(\mathbf{h})\end{array}$ & $\begin{array}{l}\bar{M}_{i}^{D} \\
\text { (h) }\end{array}$ & $\begin{array}{c}\bar{P}_{i} \\
\text { (MW) }\end{array}$ & $\begin{array}{c}\underline{P}_{i} \\
(\mathrm{MW})\end{array}$ & $\begin{array}{c}\bar{R}_{i}^{U} \\
\text { (MWh/h) }\end{array}$ \\
\hline Wind & 6 & 105000 & 1750 & 0 & 0 & 0 & 0 & 100 & 0 & 0 & 1000 & 0 & 1000 \\
\hline PV & 6 & 45000 & 1000 & 0 & 0 & 0 & 0 & 100 & 0 & 0 & 600 & 0 & 600 \\
\hline Nuclear & 4 & 140600 & 8750 & 2.04 & 3.43 & 10.49 & 16.5 & 100 & 4 & 3 & 1800 & 650 & 900 \\
\hline Coal & 4 & 86000 & 3970 & 4.25 & 2.22 & 8.8 & 23.8 & 100 & 2 & 3 & 1800 & 870 & 1080 \\
\hline CCGT & 4 & 99900 & 10390 & 6.43 & 5.81 & 7.05 & 58.5 & 100 & 2 & 2 & 1200 & 300 & 960 \\
\hline OCGT & 4 & 76000 & 9980 & 14.7 & 5.81 & 10.85 & 99.4 & 100 & 1 & 1 & 1200 & 120 & 960 \\
\hline
\end{tabular}




\section{B Aggregation Technique and Aggregated Problem}

This section provides further details on the Exhaustive Search (ES) aggregation technique which is applied in the case study. An outline of the algorithm is provided in Procedure 3. Recall from Section 4.1 that $L_{r e s}^{h}=$ $D^{h}-W^{h} \cdot C_{W}-P V^{h} \cdot C_{P}$, where $\mathrm{D}$ is demand, $\mathrm{W}$ is wind and $C_{W}$ and $C_{P}$ are the assumed maximal capacities of wind and $\mathrm{PV}$ in the system, respectively. Moreover the algorithm introduces the Normalized Root Mean Square Error (NRMSE) which is calculated as follows;

$$
\text { NRMSE }=\frac{\sqrt{\sum_{t \in T}\left(R L D C_{t}-\overline{R L D C}_{t}\right)^{2}}}{|T|},
$$

where $R L D C_{t}$ is the original RLDC, $\overline{R L D C}_{t}$ is the approximated RLDC and $|T|$ is the amount of hours in the original instance ( 8736 hours in our case study). We furthermore introduce $\chi \in X$ as a week $\chi$ belonging to the set of all weeks $X$. In our case study we selects 4 weeks out of 52 weeks, corresponding to a $92 \%$ data reduction. How this affects the Mathematical Problem Formulation is seen in Table 6. Some statistical relations between the non-aggregated and aggregated input time series are seen in Table 7. Figure 12 illustrates how the input time series differ among the three problem instances $P_{2014}, P_{2015}$ and $P_{2016}$, which similarly is seen for the aggregated time series in Figure 13.

Table 6: Relation between non-aggregated and aggregated problem sizes (Notice, these relate to $\mathrm{P}$ and not to $\mathrm{Q}$ problems). The mathematical model size is equal for the three different problems $P_{2014}, P_{2015}$ and $P_{2016}$, wherefore only one non-aggregated and one aggregated problem size are seen. The solution time, however, differs for the three instances and the listed solution times therefore relate to the average values.

\begin{tabular}{|l|ccccc|}
\multicolumn{1}{|c}{ Model } & Constraints & Variables & Non-Zero entries & Discrete Variables & Solution Time \\
\hline Non-Aggregated & 1.441 .409 & 1.013 .405 & 3.878 .673 & 524.188 & 4.2 hours \\
Aggregated & 110.871 & 77.981 & 298.301 & 40.348 & $42 \mathrm{sec}$ \\
\hline
\end{tabular}

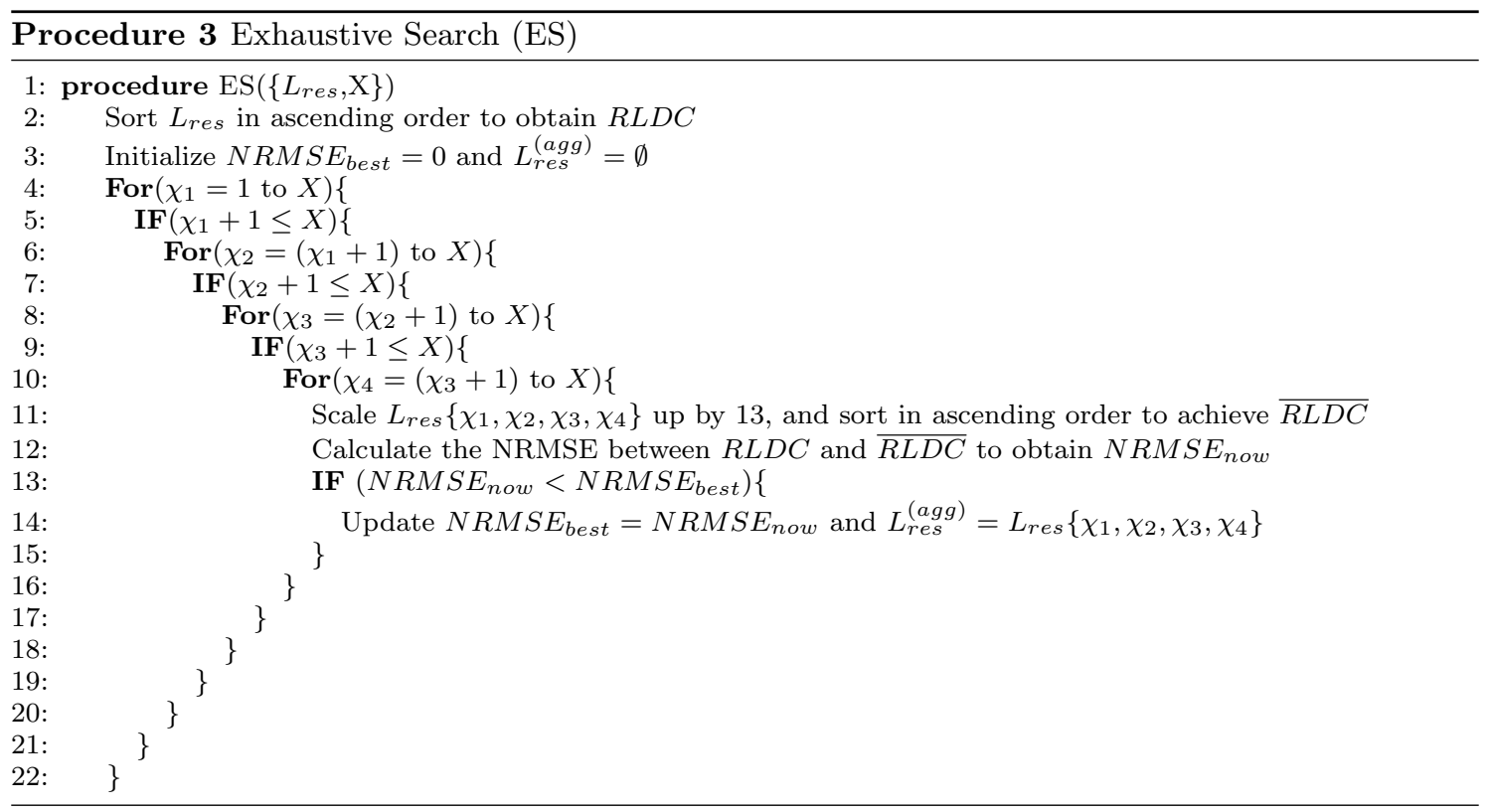




\section{Input Data}

The non-aggregated input time series consist of hourly demand profiles and hourly wind and PV availability profiles. Each problem $\left\{P_{2014}, P_{2015}, P_{2016}\right\}$ covers a single region and hence is associated to a single input time series of each type. Graphical illustrations of the non-aggregated input time series are seen in Figure 12, while the corresponding aggregated profiles are illustrated in Figure 13. To further illustrate differences among time series of the different years and differences among aggregated and non-aggregated time series, a selection of statistical measures for each profile are seen in Table 7.

Table 7: Statistical measures of the three types of input time series; demand, wind and PV both in non-aggregated and aggregated forms. Values are seen for each of the three years; 2014, 2015 and 2016. Sum relates to the cumulative values throughout the period (MWh), while Mean, Max and Min relate to the single hour values $(\mathrm{MWh} / \mathrm{h})$. Wind and PV are illustrated as predicted capacities meaning that the values indicate the wind and PV capacities arising from an investment in all candidate units of the respective type.

\begin{tabular}{|c|c|c|c|c|c|c|c|c|c|}
\hline \multirow[b]{2}{*}{ Profile } & \multirow[b]{2}{*}{ Year } & \multicolumn{4}{|c|}{ Non-Aggregated Time Series } & \multicolumn{4}{|c|}{ Aggregated Time Series } \\
\hline & & Sum & Mean & Max & Min & Sum & Mean & $\operatorname{Max}$ & Min \\
\hline \multirow{3}{*}{ Demand } & 2014 & 33.357 .780 & 3818 & 2296 & 6033 & 2.650 .526 & 3944 & 2398 & 5646 \\
\hline & 2015 & 33.526 .849 & 3838 & 2275 & 5741 & 2.700 .963 & 4019 & 2370 & 5670 \\
\hline & 2016 & 33.802 .803 & 3869 & 2213 & 6115 & 2.663 .858 & 3964 & 2642 & 5409 \\
\hline \multirow{3}{*}{ Wind } & 2014 & 15.997 .033 & 1831 & 0 & 5455 & 1.342 .075 & 1997 & 11 & 5087 \\
\hline & 2015 & 16.621 .458 & 1903 & 0 & 5267 & 1.417 .248 & 2109 & 41 & 5130 \\
\hline & 2016 & 14.444 .281 & 1653 & 0 & 5333 & 1.182 .706 & 1760 & 42 & 5182 \\
\hline \multirow{3}{*}{ PV } & 2014 & 4.313 .297 & 384 & 0 & 3500 & 208.275 & 310 & 0 & 3152 \\
\hline & 2015 & 4.263 .148 & 488 & 0 & 3500 & 287.205 & 427 & 0 & 3359 \\
\hline & 2016 & 4.273 .258 & 489 & 0 & 3500 & 352.316 & 524 & 0 & 3477 \\
\hline
\end{tabular}



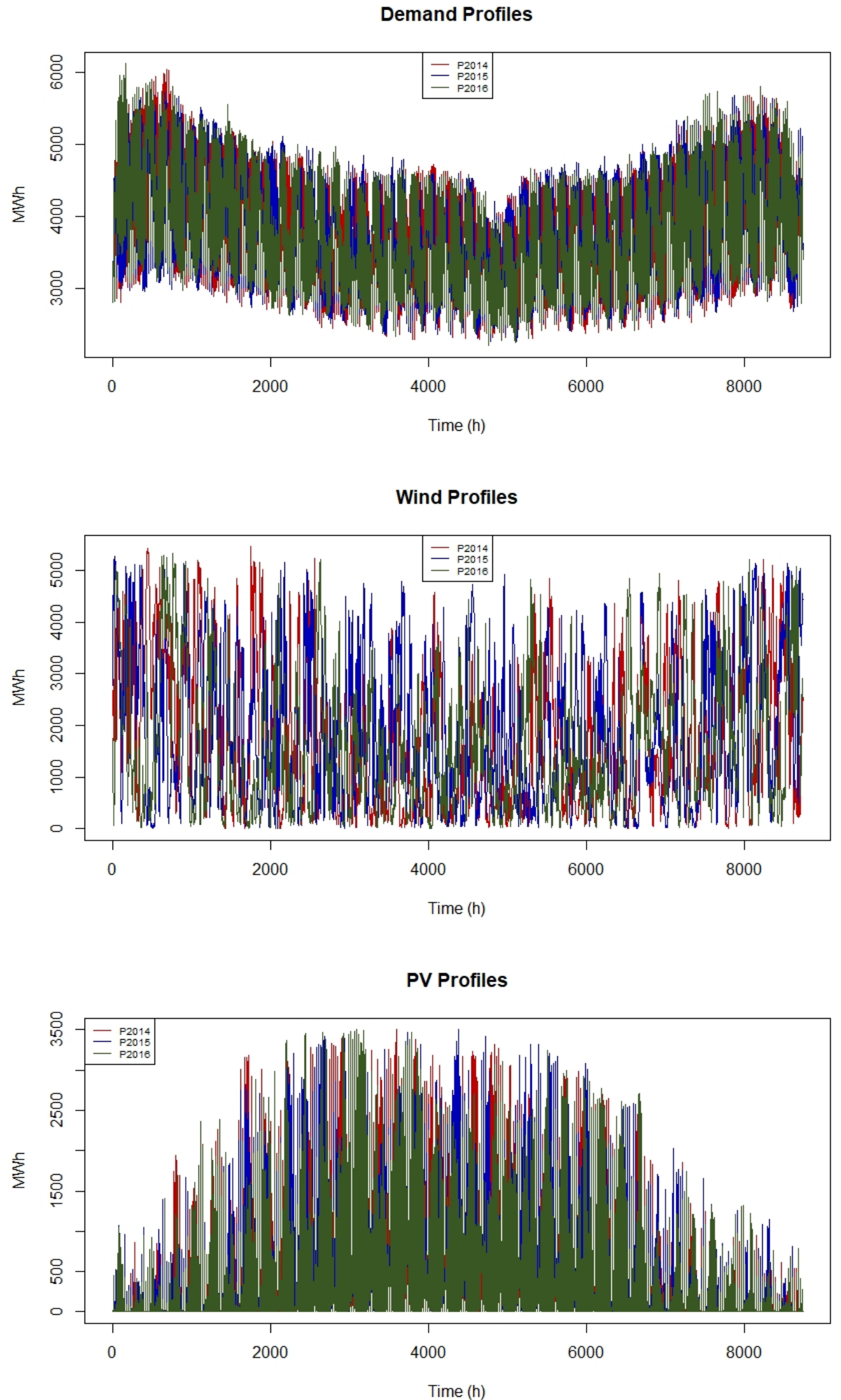

Fig. 12: Graphical illustration of how the input time series differ for the three years 2014, 2015 and 2016. Wind and PV are illustrated as predicted capacities throughout the year. This means that the graphs illustrate the wind and PV capacities arising from an investment in all candidate units of the respective type. 
Aggregated Demand Profiles

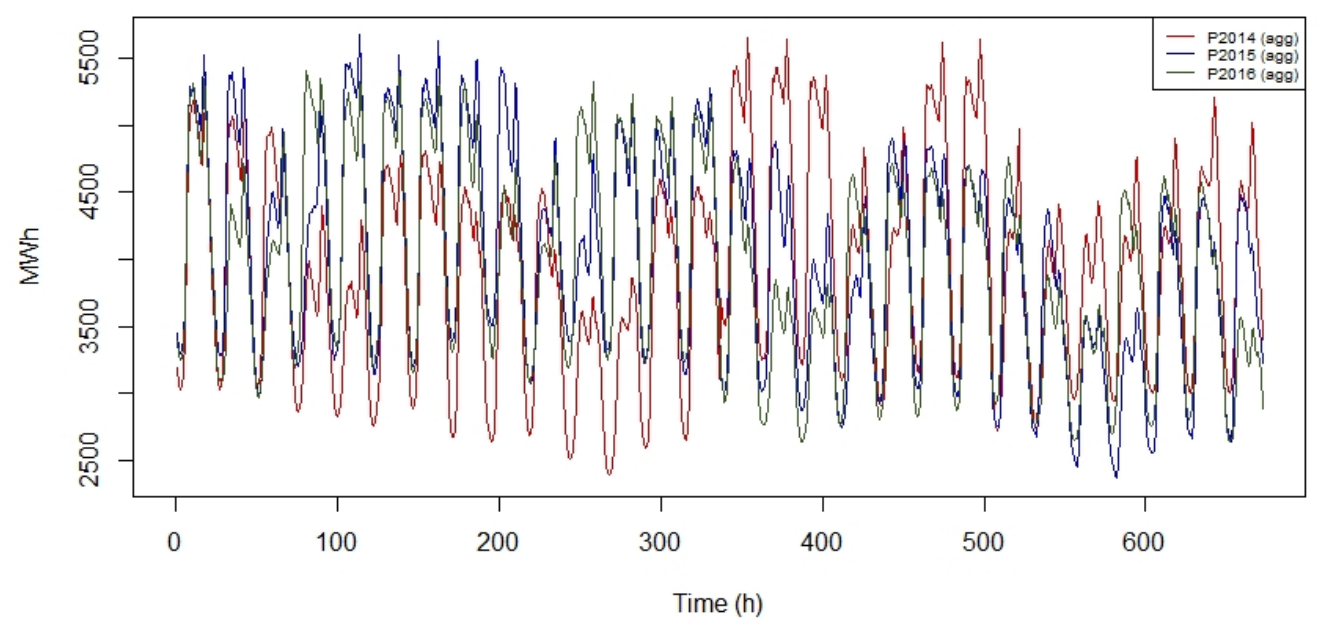

Aggregated Wind Profiles

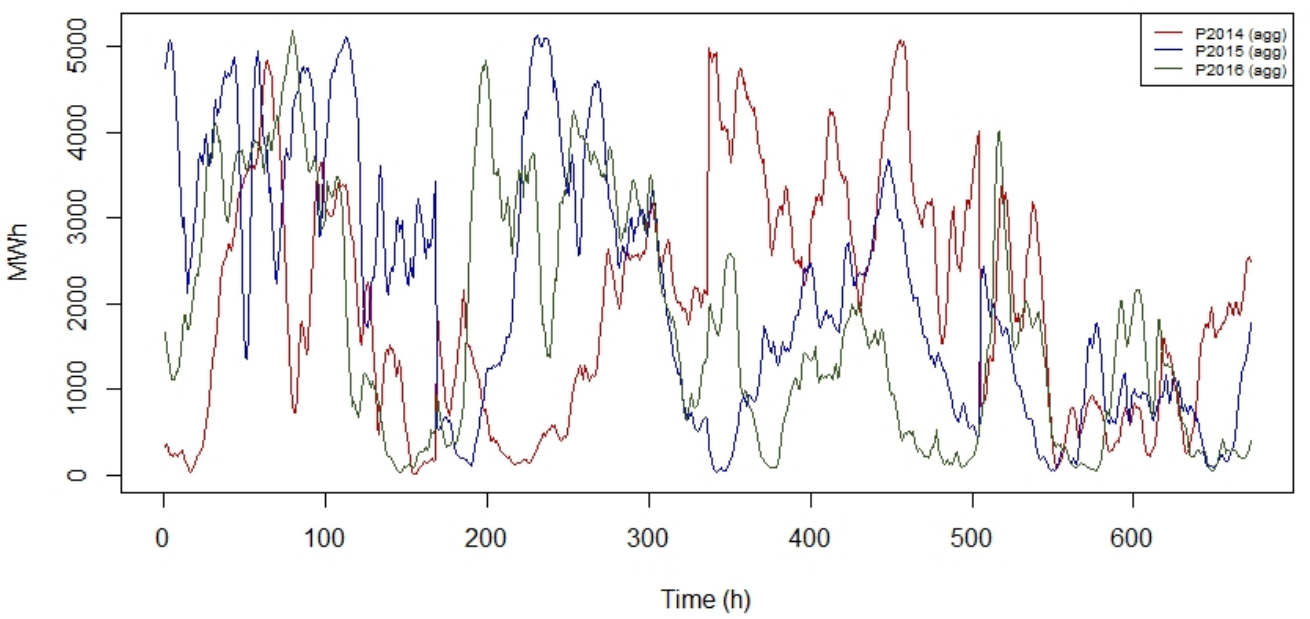

Aggregated PV Profiles

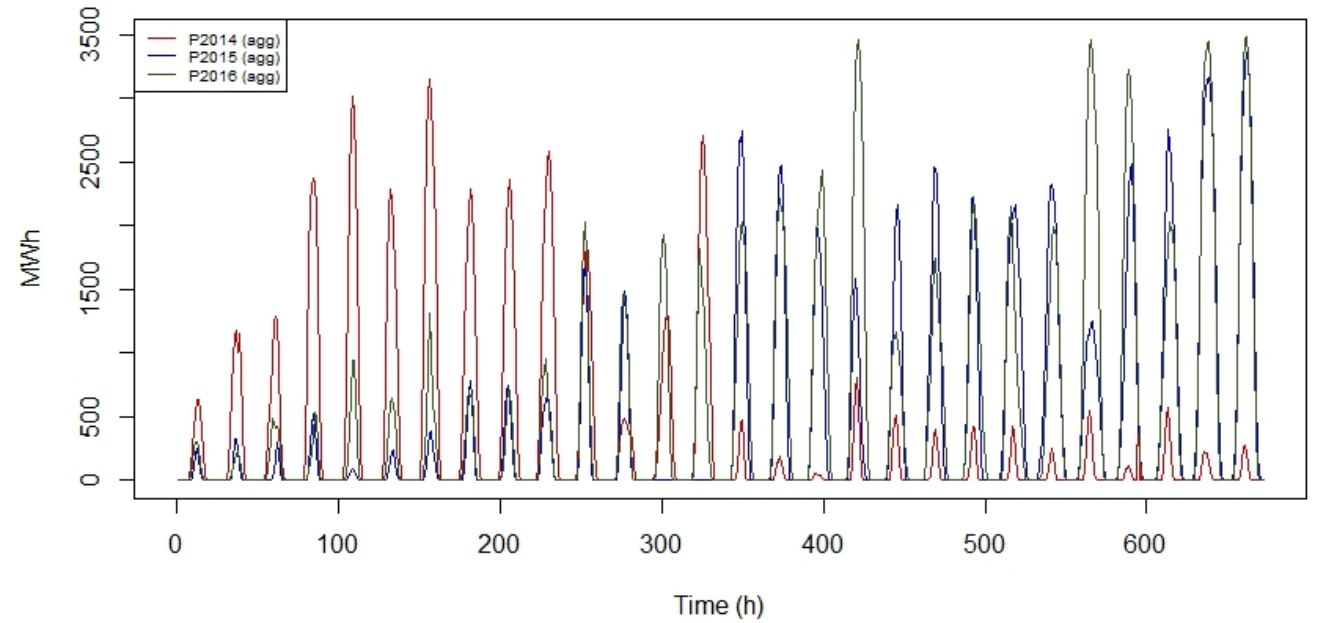

Fig. 13: Graphical illustration of how the aggregated input time series differ for the three years 2014, 2015 and 2016. Wind and PV are illustrated as predicted capacities throughout the aggregated period. This means that the graphs illustrate the wind and PV capacities arising from an investment in all candidate units of the respective type. 


\section{Supplementary Graphs for the Result Section}
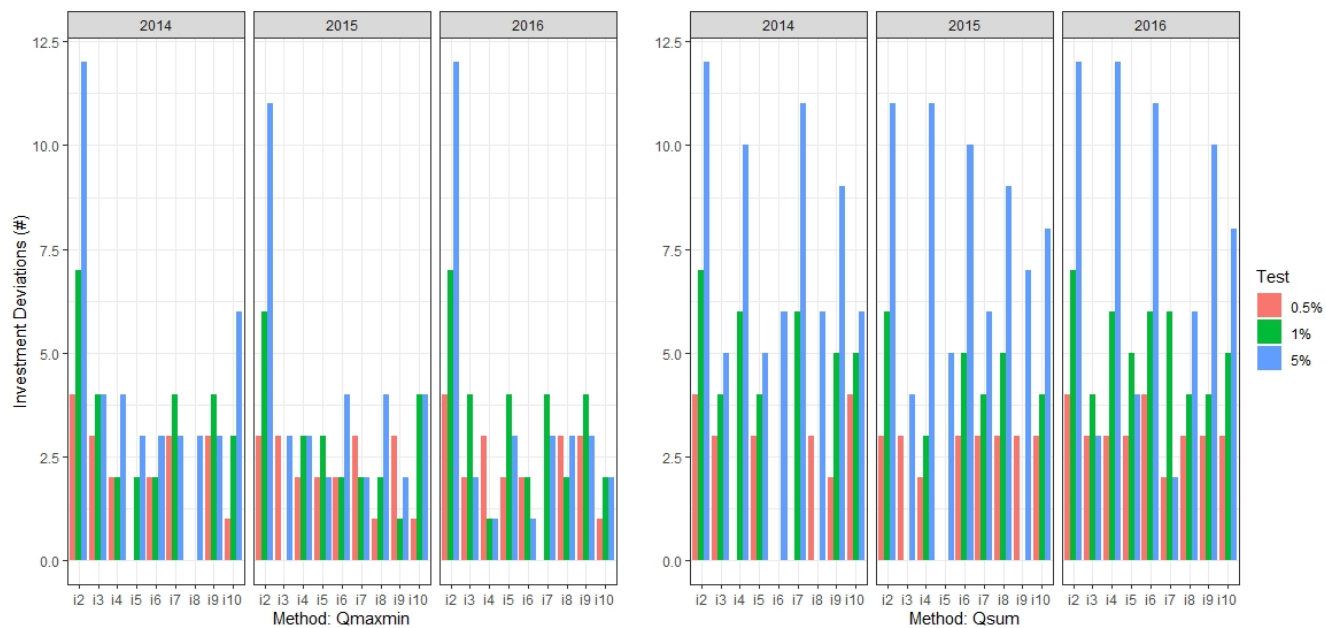

Fig. 14: The evolution of the deviation in investment strategies across the iterations. As the method prevents the occurrence of identical solutions, missing columns are caused by non-existing solutions. 


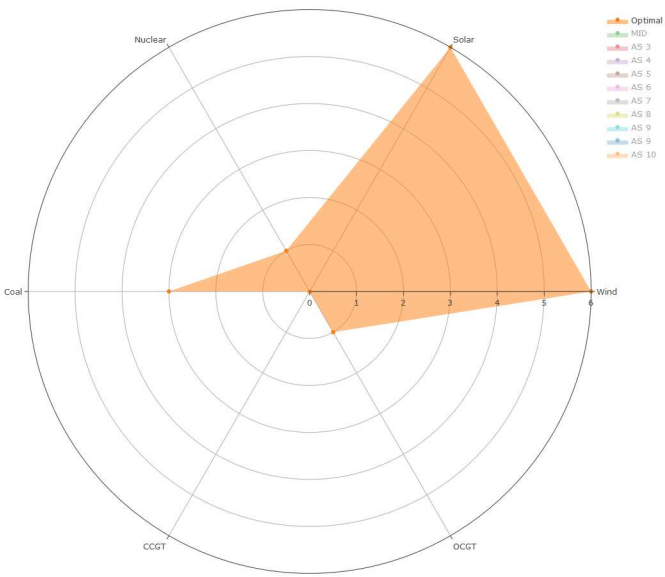

(a) Optimal solution

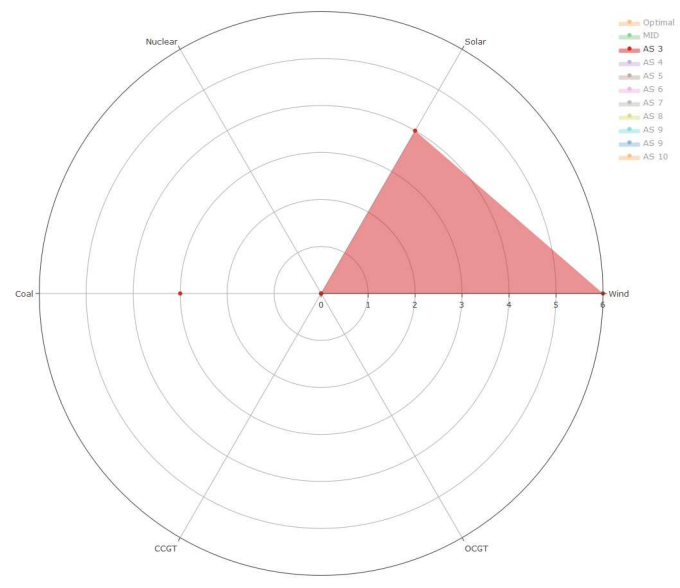

(c) Alternative solution (iteration 3)

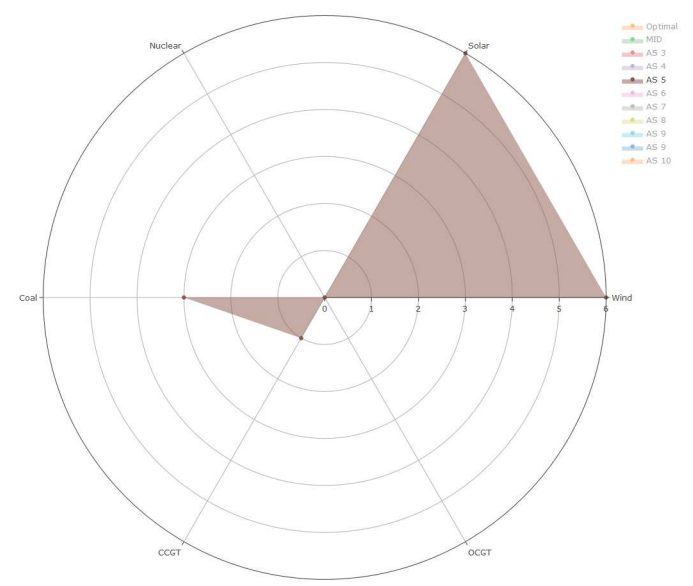

(e) Alternative solution (iteration 5)

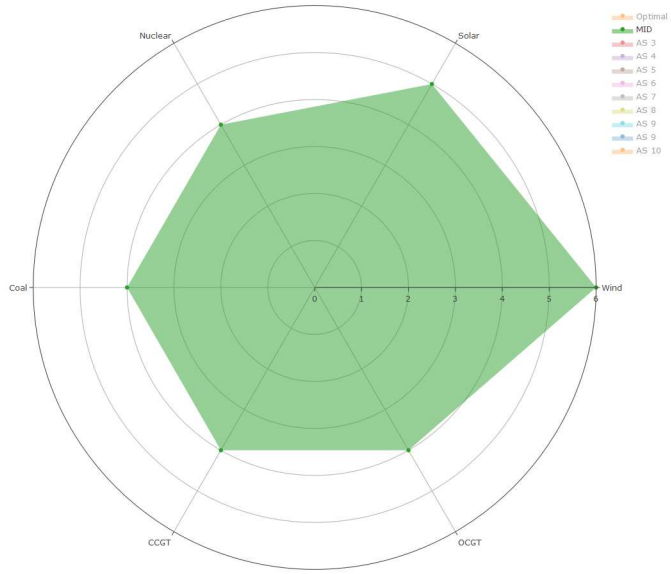

(b) Maximum Investment Different Solution

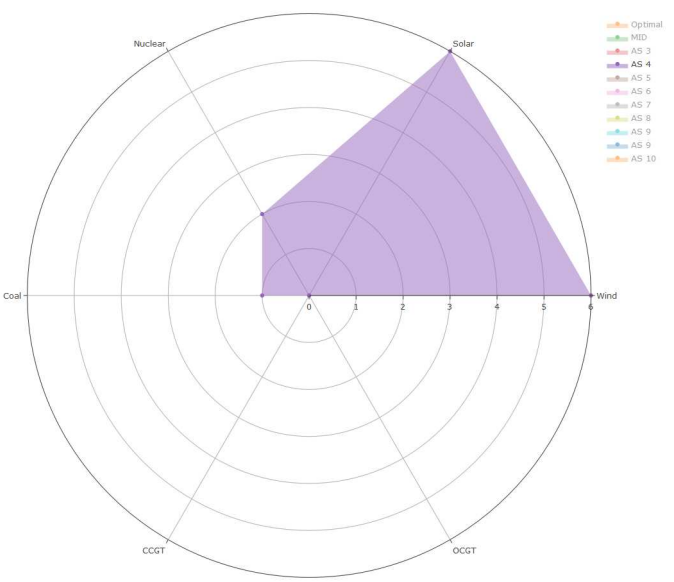

(d) Alternative solution (iteration 4)

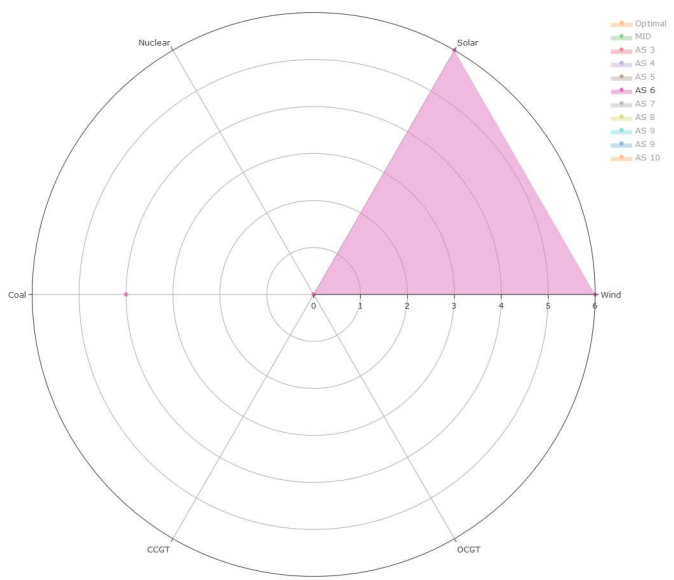

(f) Alternative solution (iteration 6)

Fig. 15: Investment decisions for each iteration of the PoMDS algorithm solving the $Q_{\operatorname{maxmin}}$ problem. The solutions are related to $P_{2014}$ and a maximum deviation in system costs of $5 \%$. 


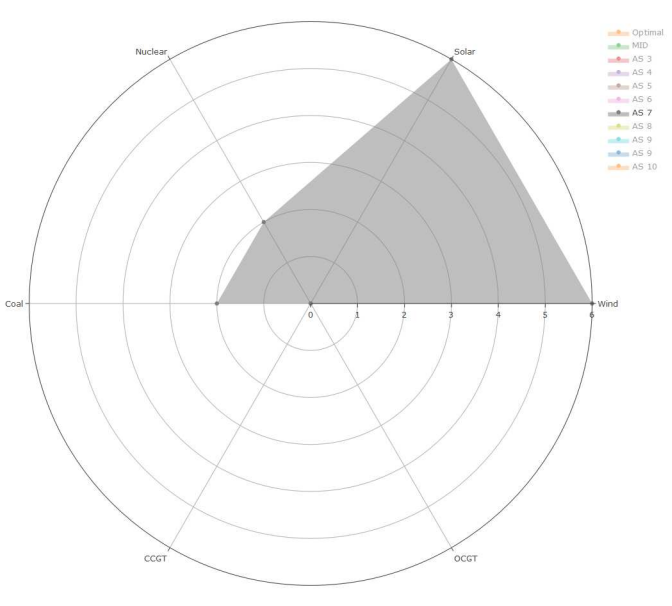

(g) Alternative solution (iteration 7)

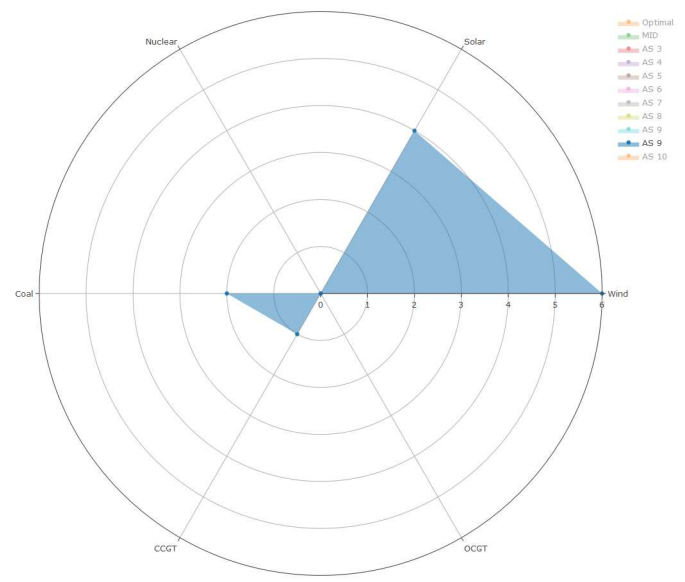

(i) Alternative solution (iteration 9)

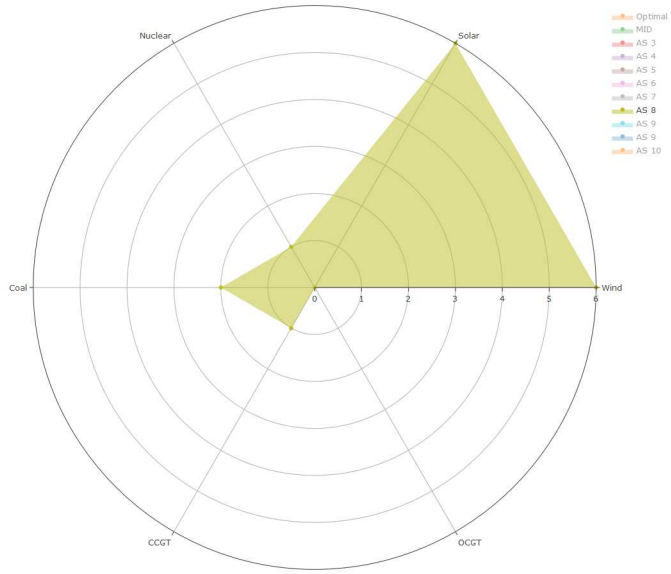

(h) Alternative solution (iteration 8)

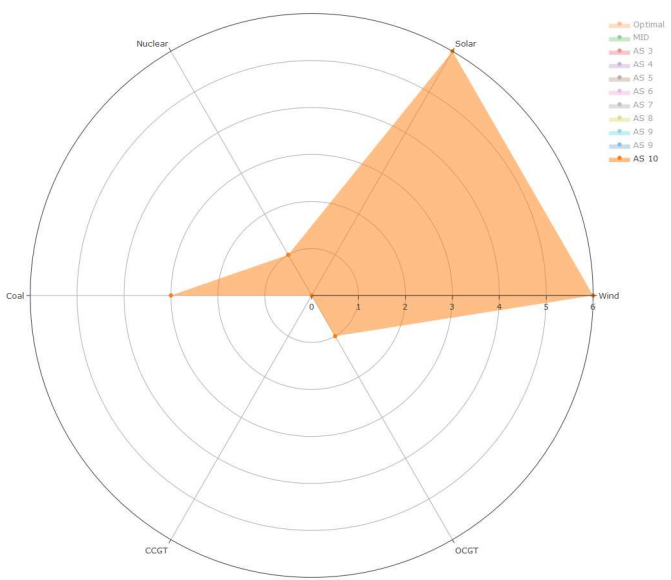

(j) Alternative solution (iteration 10)

Fig. 15: (Continued) Investment decisions for each iteration of the PoMDS algorithm solving the $Q_{\text {maxmin }}$ problem. The solutions are related to $P_{2014}$ and a maximum deviation in system costs of $5 \%$. 


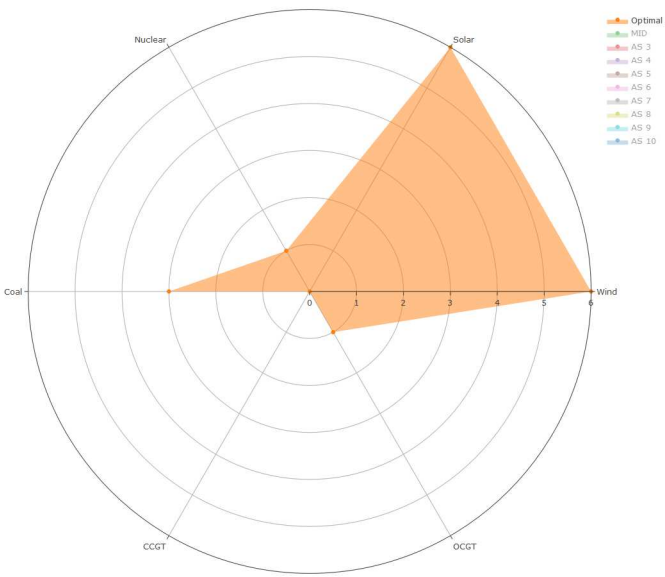

(a) Optimal solution

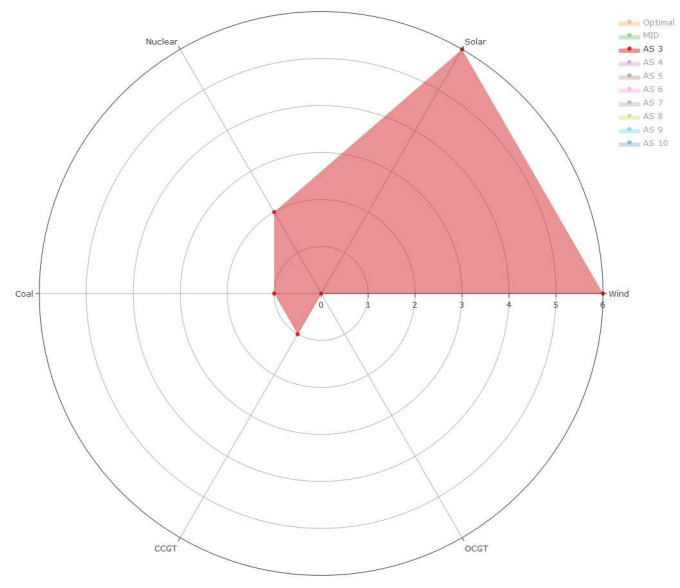

(c) Alternative solution (iteration 3)

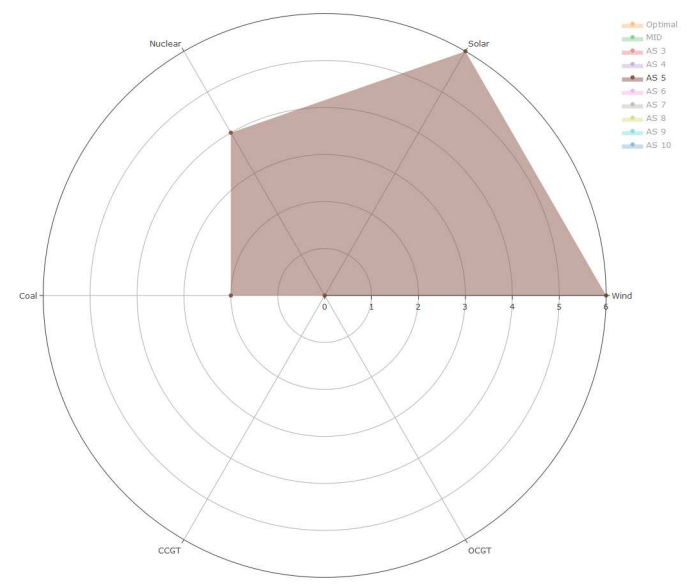

(e) Alternative solution (iteration 5)

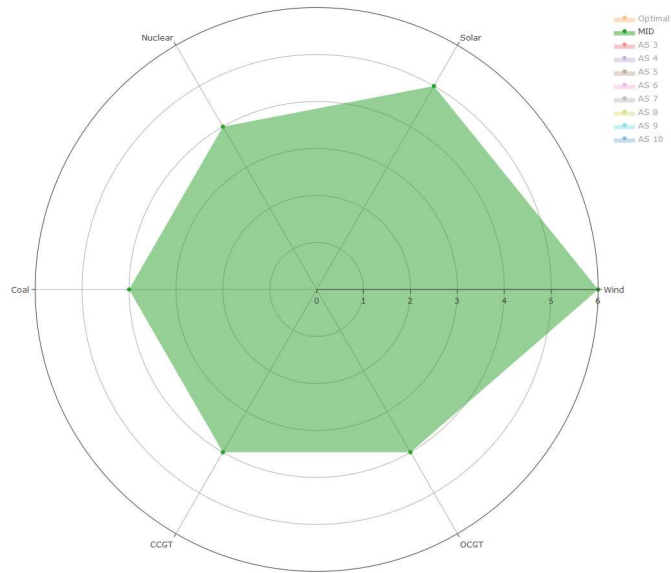

(b) Maximum Investment Different Solution

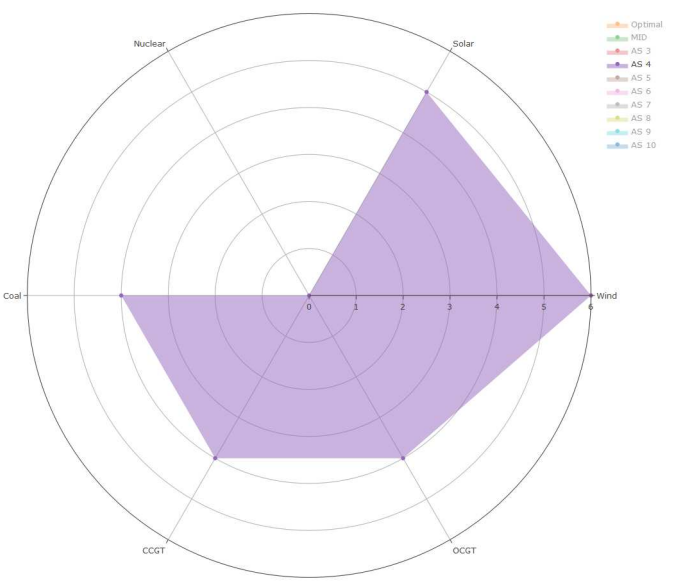

(d) Alternative solution (iteration 4)

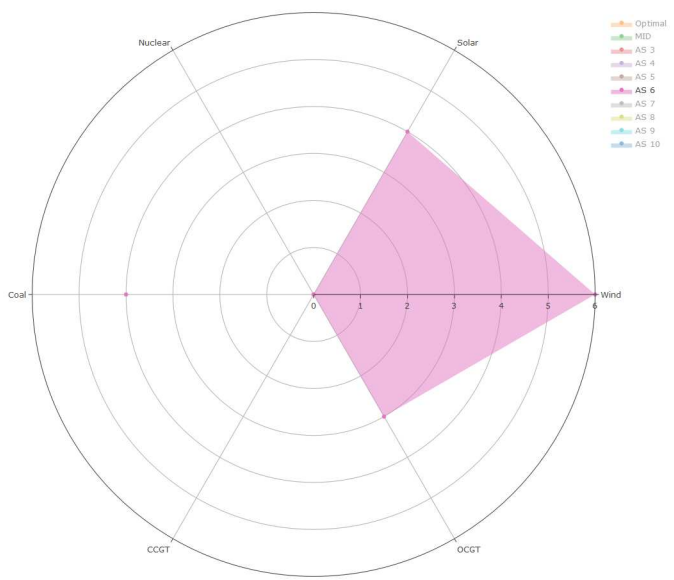

(f) Alternative solution (iteration 6)

Fig. 16: Investment decisions for each iteration of the PoMDS algorithm solving the $Q_{\text {sum }}$ problem. The solutions are related to $P_{2014}$ and a maximum deviation in system costs of $5 \%$. 


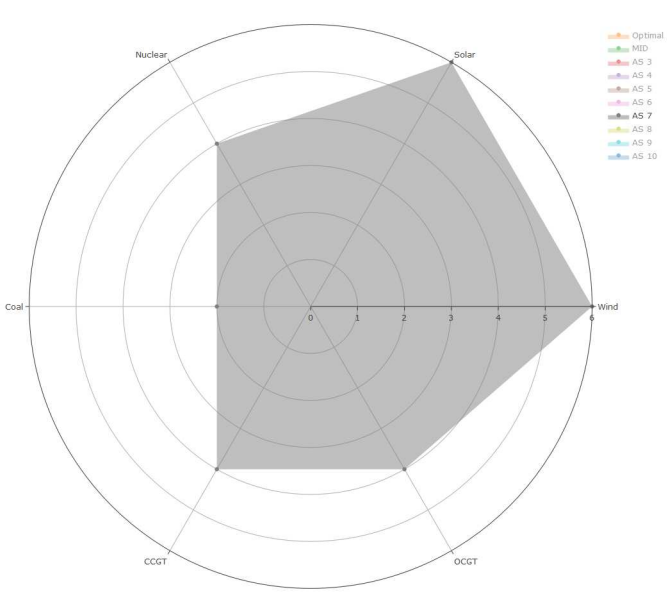

(g) Alternative solution (iteration 7)

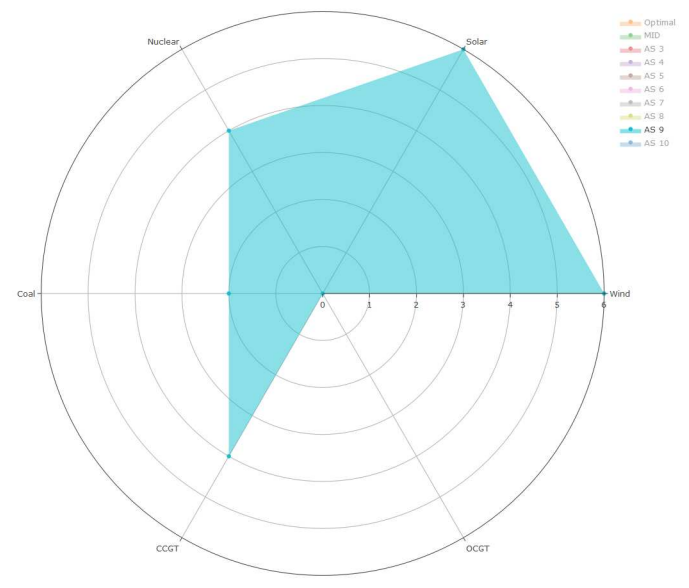

(i) Alternative solution (iteration 9)

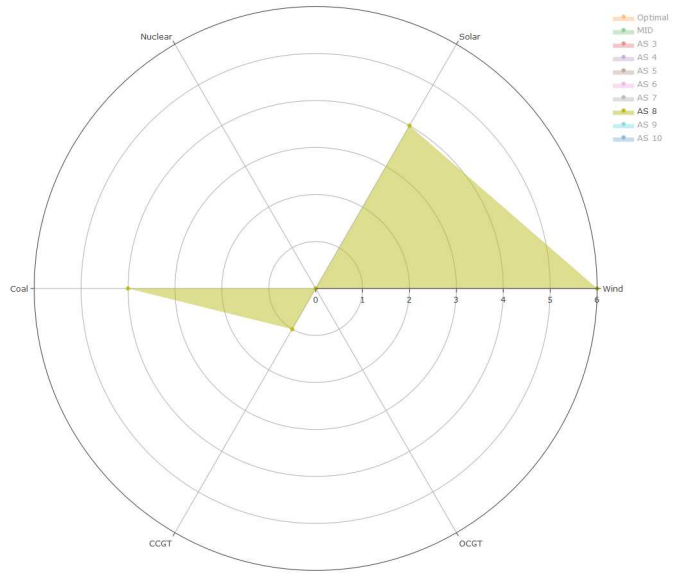

(h) Alternative solution (iteration 8)

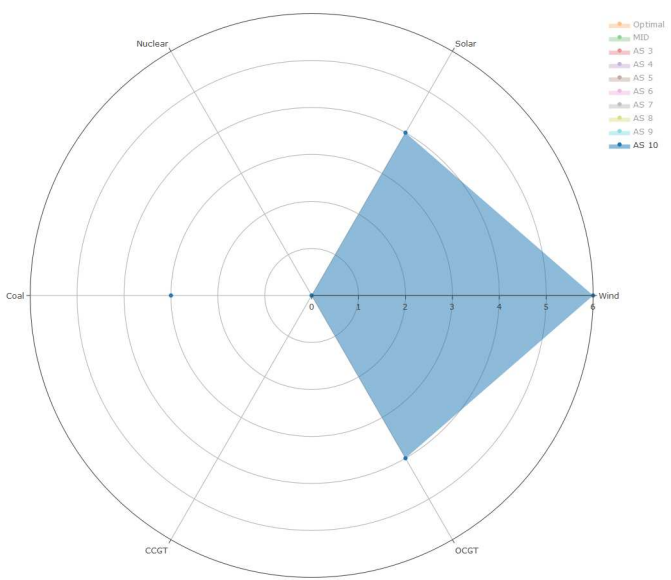

(j) Alternative solution (iteration 10)

Fig. 16: (Continued) Investment decisions for each iteration of the PoMDS algorithm solving the $Q_{\text {sum }}$ problem. The solutions are related to $P_{2014}$ and a maximum deviation in system costs of $5 \%$.

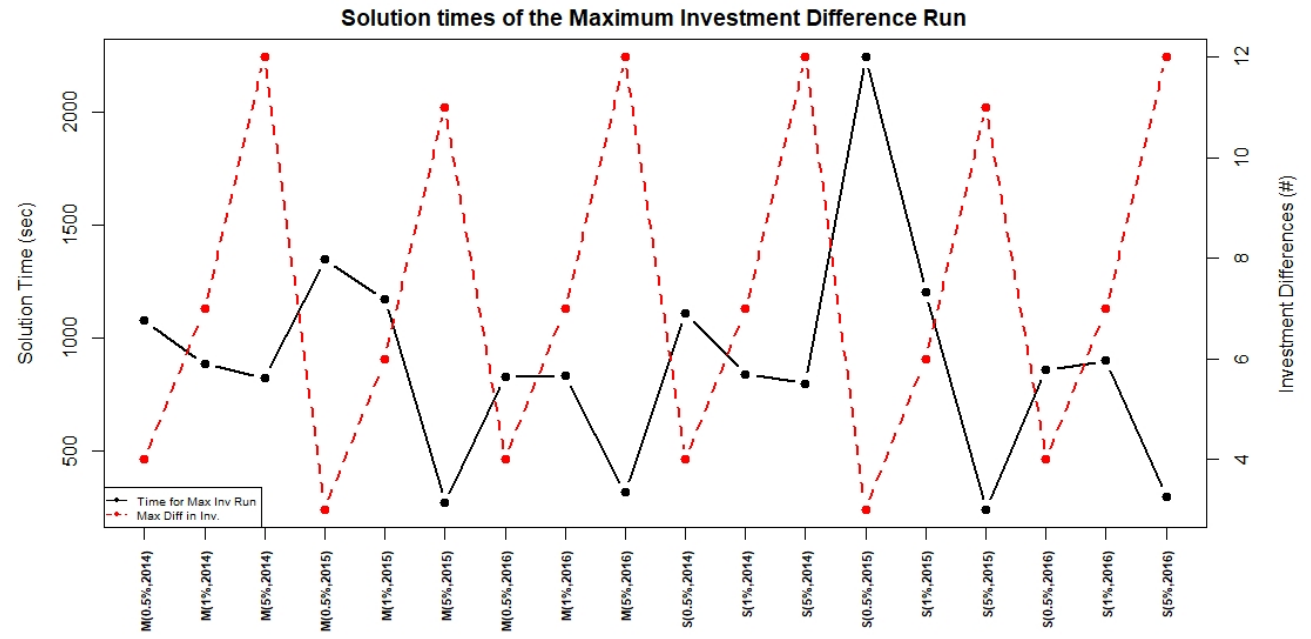

Fig. 17: Illustration of the solutions being the most different from the optimal one with respect to investment decisions. The relation between the degree of difference and the associated solution time is seen. 